\title{
Experimental Supersymmetry
}

Richard L Amoroso (https://orcid.org/0000-0003-2405-9034)

Noetic Advanced Studies Institute;

amoroso@noeticadvancedstudies.us

Received: 3 November 2020 Accepted: Published:

\begin{abstract}
A radical, albeit pragmatic protocol for experimental access to putative String/M-theoretic, Einstein Unified Field Mechanical (UFM) additional dimensionality (XD) of the brane-bouquet bulk is presented. If successful, results demonstrate the existence dimensionality beyond the metric of observed physical reality provided by the Standard Model (SM) of particle physics and Cosmology. Quantum Mechanics (QM), as well-known is incomplete and further, should no longer be considered the basement of reality; meaning, Locality and Unitarity - the fundaments of quantum theory are an insufficient basis for extending the representation of reality. A seminal model of Tight Bound States (TBS) below the lowest Bohr orbit in the hydrogen atom, proposed by Vigier, is extended to a KaluzaKlein-like (KK) cyclical tier of XD hyperspherical cavities defined within a manifold of uncertainty (MOU) of finite radius up to the semi-quantum limit, predicting, within its domain, new spectral lines in Hydrogen. The proposed protocol for this process, with phase modifications, provides efficacy of large-scale additional dimensionality (LSXD) of the brane bulk; XD-LSXD incursion duality accesses nonlocal Einsteinian UFM phenomena, leading to myriad new classes of technological innovation.
\end{abstract}

Physis kryptesthai philei; Nature hides itself - Heraclitus, 500 B.C.E.

Keywords: Dirac hypertube; Dirac polarized vacuum; M-theory; manifold of uncertainty; nonlocality; semi-quantum limit; supersymmetry; tight bound states, unified field theory

\section{Introductory Precis / Motivation}

A concatenation of generally overlooked theoretical approaches relative to current thinking is utilized to formulate a protocol for the experimental demonstration of additional dimensionality (XD) of a brane-bouquet Bulk [1] within which observed/realized 3D space is a restricted brane inside this bulk which phenomenologically entails a Kaluza-Klein-like cyclic duality between the two: 1) compact XD up to a semi-quantum domain wall limit as manifest by the uncertainty principle and 2 ) large-scale additional dimensionality (LSXD) with likely infinite extension into a multiverse $[2,3]$. This local-nonlocal duality can be envisioned in terms of the Dirac electron hypertube model [4,5].

While a dramatic advance suggesting an imminent paradigm shift if successful, since technological innovation is not inherent in this basic TBS spectroscopic protocol, discussion is included on the theoretical framework for required modifications to implement assumed myriad technological innovations. That scenario requires an advanced form of universal quantum computing (UQC) with a dual XD/LSXD UFM/M-theoretic topological phase qubit [6] to program the spacetime vacuum utilizing both sides of the Cellular Least Units (CLU) Dirac hypertubes tessellating the polarized covariant vacuum of the Dirac type [2-9]. 
Initial motivation for the protocol design arose from seminal work by Vigier on Tight-bound States (TBS) in the hydrogen atom [10-12]. The premise of TBS below the lowest Bohr orbit, was myopically ignored initially as logically untenable because an atomic electron could only have specific defined energies, $E_{n}=-\left(h c R_{\infty} / n^{2}\right)$, where $R_{\infty}$ is the Rydeberg constant, $n \in\{1,2,3 \ldots\}$ and at $n=1$, an electron could not be any closer to the nucleus! Twelve years later after developing a holographic multiverse cosmology [2,3]; the profundity of indicia TBS provided was finally realized as the key to low energy tabletop brane topological cross section (sans supercollider particle sprays) experimental protocols able to surmount the finite domain wall manifold of quantum uncertainty.
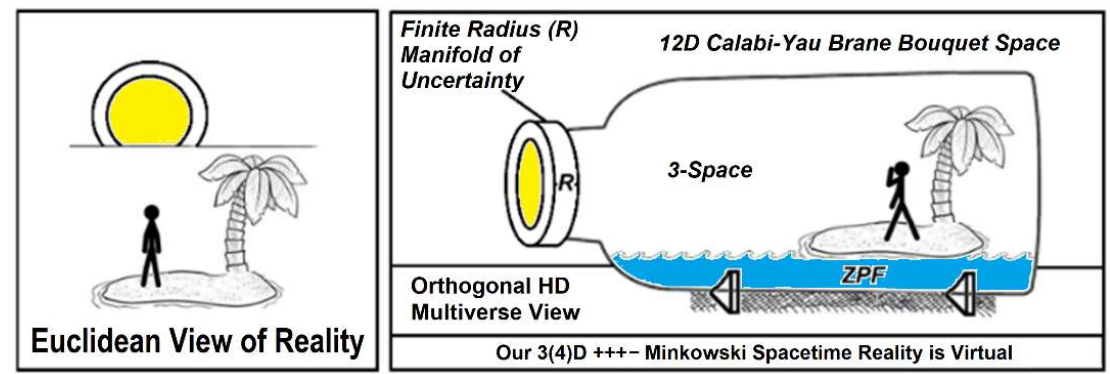

Figure 1. Beyond current 3D Standard Model (SM) spacetime lies LSXD Hidden by a finite hypertube domain wall of uncertainty. a) View of current 3D SM reality. b) Duality of XD and nonlocal LSXD brane world.

Figure $1 \mathrm{~b}$ ) symbolically suggests the regime of the proposed TBS protocol predicting new spectral lines within a finite radius Manifold of Uncertainty (MOU) up to the semi-quantum limit, the local end of a wormhole/Dirac hypertube within which is a hyperspherical tier of putative supersymmetric (or mirror symmetric) XD QED cavities (CQED), each with its own spectral line. Note that at the MOU limit (like an atomic electron blowing off to infinity when sufficient energy is achieved for escape); no additional MOU spectral lines will be discovered; as entry to infinite size LSXD of the brane bouquet bulk topology is suggested to occur.

\section{Tight Bound State (TBS) Modeling - Indicia of XD QED Hyperspherical Cavities}

Tight Bound States (TBS) due to electromagnetic interactions at small distances below the lowest Bohr orbit have been postulated for the Hydrogen atom [10-15]. Summarizing seminal work of Vigier - in the usual understanding of atomic physics spin-orbit and spin-spin coupling perturbations for example, give rise to only small corrections in classical Bohr energy levels. However, with distances in the $1 / \mathrm{r}^{3}$ and $1 / \mathrm{r}^{4}$ range these interaction terms, until now overlooked, could be much higher than the Coulomb term at distances much less than the Bohr radius - predicting indicia of new physics $[10,13]$. Corben [14] noticed motion of a point charge in a magnetic dipole field at rest is highly relativistic with orbits of nuclear dimensions. Further investigation undertaken by $[10-13,15]$ represented an extension of the Pauli equation to a two-body system as defined by the Hamiltonian

$$
H=\frac{1}{2 m_{1}}\left(\vec{P}_{1}-e_{1} \vec{A}\left(\vec{r}_{1}\right)\right)^{2}+\frac{1}{2 m_{2}}\left(\vec{P}_{2}-e_{2} \vec{A}\left(\vec{r}_{2}\right)\right)^{2}+\frac{1}{4 \pi \varepsilon_{0}} \frac{e_{1} e_{2}}{\left|\vec{r}_{1}-\vec{r}_{2}\right|}+V_{d d}
$$

where, $m_{i}$ is mass, $\vec{P}_{i}$ momentum, $e_{i}$ charge, $\vec{r}_{i}$ position of the particles $(i=1,2), \vec{A}$ is the electromagnetic vector potential and $V_{d d}$ the dipole-dipole interaction term:

$$
V_{d d}=-\left(\frac{\mu_{0}}{4 \pi}\right) \vec{\mu}_{1} \vec{\mu}_{2} \delta\left(\vec{r}_{1}-\vec{r}_{2}\right)+\left(\frac{\mu_{0}}{4 \pi}\right)\left[\frac{\vec{\mu}_{1} \vec{\mu}_{2}}{\left|\vec{r}_{1}-\vec{r}_{2}\right|^{3}}-\frac{3\left[\vec{\mu}_{1}\left(\vec{r}_{1}-\vec{r}_{2}\right)\right] \cdot\left[\vec{\mu}_{2}\left(\vec{r}_{1}-\vec{r}_{2}\right)\right]}{\left|\vec{r}_{1}-\vec{r}_{2}\right|^{5}}\right] .
$$

In the center-of-mass frame and with a normal magnetic moment, $\vec{\mu}=(e / m) \vec{S}$ the Hamiltonian (2) 
becomes:

$$
\begin{aligned}
& H=\frac{1}{2 m_{1}} p^{2}-\left(\frac{\mu_{0}}{4 \pi}\right) \frac{e_{1} e_{2}}{m_{1} m_{2}} \frac{\vec{S} \vec{L}}{r^{3}}+\left(\frac{\mu_{0}}{4 \pi}\right)^{2} \frac{e_{1}^{2} e_{2}^{2} \hbar^{2}}{4 m_{1} m_{2} m} \frac{1}{r^{4}}+ \\
& \frac{1}{4 \pi \varepsilon_{0}} \frac{e_{1} e_{2}}{r}-\left(\frac{\mu_{0}}{4 \pi}\right) \frac{e_{1} e_{2}}{m_{1} m_{2}} \vec{s}_{1} \vec{s}_{2} \delta(\vec{r})+\left(\frac{\mu_{0}}{4 \pi}\right) \frac{e_{1} e_{2}}{m_{1} m_{2}}\left[\frac{\vec{s}_{1} \vec{s}_{2}}{r^{3}}-\frac{3\left(\vec{s}_{1} \vec{r}\right) \cdot\left(\vec{s}_{2} \vec{r}\right)}{r^{5}}\right],
\end{aligned}
$$

where $r, p, \vec{S}, \vec{L}$ relate to relative motion and $m$ is a reduced mass. The usual Pauli approximation producing (3) is improved by keeping an energy term in the Hamiltonian since $m$ is of the resonant energy order of interest. This new Hamiltonian depends on energy through the effective mass, $m^{*}$ as in, $m^{*}=m+\left(E / 8 c^{2}\right)[10-13]$.

In terms of total spin angular momentum, the self-consistent Hamiltonian of the Barut-Vigier model is:

$$
\begin{aligned}
& H=\frac{1}{2 m^{*}} p^{2}+\frac{1}{4 \pi \varepsilon_{0}} \frac{e_{1} e_{2}}{r}-\left(\frac{\mu_{0}}{4 \pi}\right) \frac{e_{1} e_{2}}{8\left(m^{*}\right)^{2}} \frac{\vec{J}^{2}-\vec{L}^{2}-2 \vec{S}^{2}}{r^{3}}+\left(\frac{\mu_{0}}{4 \pi}\right)^{2} \frac{e_{1}^{2} e_{2}^{2} \hbar^{2}}{16\left(m^{*}\right)^{3}} \frac{1}{r^{4}}- \\
& \left(\frac{\mu_{0}}{4 \pi}\right) \frac{4 \pi e_{1} e_{2}}{8\left(m^{*}\right)^{3}}\left(\vec{S}^{2}-\frac{3}{2} \hbar^{2}\right) \delta(\vec{r})-\left(\frac{\mu_{0}}{4 \pi}\right) \frac{3 e_{1} e_{2} \hbar^{2}}{8\left(m^{*}\right)^{2}} \frac{Q}{r^{3}}
\end{aligned}
$$

with operator $Q=\left(1 / \hbar^{2}\right) \cdot\left((\vec{S} \vec{r})^{2} / r^{2}\right)[10,15]$.

Continuing to follow Vigier [10-13], the possibility of TBS physics as derived from Hamiltonian (4) is shown by important spin channel resonance phenomena, $S=1, L=1$ and $J=0$ because attractive spin interactions are strongest with an effective potential appearing in the radial Schrödinger equation (5) and simplified form (6) when limited to spherical terms:

$$
\begin{gathered}
\frac{d^{2} u}{d r^{2}}-\left[\frac{2 m^{*}}{\hbar^{2}} \frac{1}{4 \pi \varepsilon_{0}} \frac{e_{1} e_{2}}{r}+\frac{2}{r^{2}}+\left(\frac{\mu_{0}}{4 \pi}\right) \frac{e_{1} e_{2}}{4\left(m^{*}\right)} \frac{6}{r^{3}}+\right. \\
\left.\left(\frac{\mu_{0}}{4 \pi}\right)^{2} \frac{e_{1}^{2} e_{2}^{2}}{8\left(m^{*}\right)^{2}} \frac{1}{r^{4}}-\left(\frac{\mu_{0}}{4 \pi}\right) \frac{e_{1}^{2} e_{2}^{2}}{8\left(m^{*}\right)^{2}} \frac{\delta(r)}{r^{2}}-\frac{2 m^{*} E}{\hbar^{2}}\right] \mu(r)=0 \\
\frac{d^{2} X}{d r^{2}}+\frac{2 m}{\hbar^{2}}[E-V(r)] X=0,
\end{gathered}
$$

which contains a form for the effective potential in the inverse power law:

$$
V(r)=\frac{A}{r^{4}}+\frac{B}{r^{3}}+\frac{C}{r^{2}}+\frac{D}{r} .
$$

At large distances this potential is an attractive Coulomb tail with a repulsive core at small distances due to the $A / r^{4}$ term [11]. For proper values of potential (5) its coefficients could have another potential well in addition to the one at distances of the order of the Bohr radius where new physics is suggested to be located. Additional theoretical details on the seminal development of TBS by Vigier can be found in [10-15].

Implementing TBS experimental technology requires significant new concepts and an extension of the basis of fundamental reality. Gauge theory conditions, basis for much of modern physics, are approximations, suggesting additional theory is required to complete the Standard Model (SM). Numerous Quantum Electro Dynamic (QED) tests have discovered a range of anomalies at the $\sigma 5$ 
[16] and $\sigma 6$ levels, suggesting proximity of additional physics beyond the SM. QED tests are often based on X-ray spectroscopic measurements. Recent results along these lines come from a program by Chantler [16-18]. Several tests of diverse forms over 10 to 15 years produced many possible discrepancies in QED theory; initially, interpretation problems and critical views of possible experimental error generally left those results ignored by the physics community. This changed in 2012 when a more sophisticated experiment by Chantler's team produced more dramatic results [16]. The new QED test $(Z>20)$ was for the $w\left(1 s 2 p^{1} P_{1} \rightarrow 1 s^{21} S_{0}\right)$ x-ray resonance line transition energy in trapped Helium-like (2 electrons) Titanium $\left(\mathrm{Ti}^{20+}\right)$ ions which had a statistical significance coefficient that rose to the level of 5 standard deviations; one of the most statistically significant discrepancies from QED theory to date, for the $\left(1 s 2 p^{1} P_{1} \rightarrow 1 s^{21} S_{0}\right)$ orbital transition energy which could significantly help establish Hydrogen-like lines of highly charged ions as a new class of transfer standards in $\mathrm{x}$ ray spectroscopy $[18,19]$.

$\mathrm{xxx}$

Firstly, we suspect, but not with sufficient insight to attempt to calculate from von Neumann's postulate suggesting that speed of collapse of the quantum wavefunction [20] could be applied to explain the marked improvement between Chantler's initial two decades of experiments on hydrogen and the 2012 NIST experiment on Helium-like titanium with a 5 statistical standard deviations improvement $[16,17]$. Is it possible that the difference in radius of electron orbits between hydrogen and 2-electron titanium ions could play a part in explaining the QED violation effect or provide indicia of hidden XD/LSXD? Such a hypothesis utilizing an SM 3-space Copenhagen interpretation seems untenable. Von Neumann's conjecture (merely a statement) is not sufficiently understood to postulate, especially in conjunction with controversy regarding physicality of components of the wavefunction in relation to the mathematics of the Schrödinger equation or measurement.

However, taking license for radical suppositions, we postulate von Neumann's speed of collapse conjecture [20] is not a simple comparative Planck time/velocity calculation between the atomic radius of a hydrogen and titanium atom as might be supposed by the SM Copenhagen interpretation. In contrast, for XD-LSXD duality in the context of a periodic Kaluza-Klein (KK) cyclicality and the Dirac electron hypertube $[4,5]$ the scenario could be dramatically different.

The Planck length, $\ell_{P}=\sqrt{G \hbar / c^{3}} \approx 10^{-35} \mathrm{~m} / \mathrm{s}$ conjectured where quantum zero-point oscillations distort Euclidean spherical geometry as a spacetime foam with $(c / \ell)=(E / \hbar)$ the order of the oscillation frequency, suggested to determine the degree of deviation from Euclidean geometry.

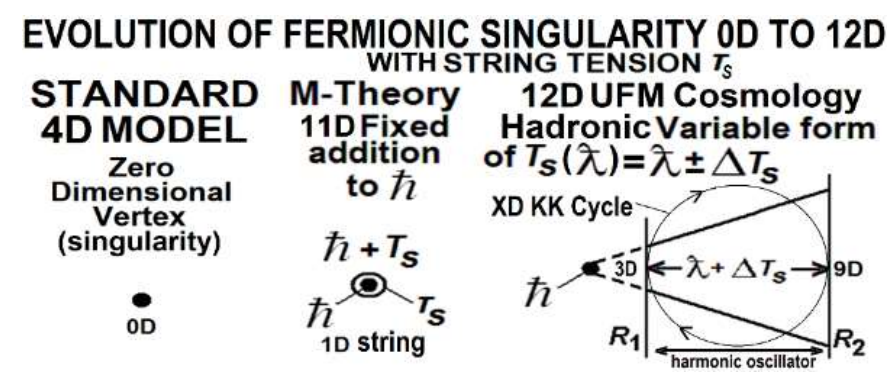

Figure 2. a) Usual 3D SM consideration of a fermion (fundamental physical object) as a 0D singularity. b) Current $1 \mathrm{D}$ basis of string theory, a fixed length string tension $T_{S}$ added to the Planck length, $\hbar+T_{S}$. c) UFM M-theoretic model reverting to the original hadronic form of string theory with variable $T_{S}$, to include continuous-state KKlike cyclical compactification, $T_{S}=\lambda+\Delta T_{0}$, utilizing $\lambda$, original Stoney, electromagnet precursor to $\hbar$.

Figure 2 attempts to illustrate why a modified M-theoretic/UFM model works better by reverting to the original hadronic form of $T_{S}$, as clarified below in terms of Cramer's Transactional Interpretation, continuous-state dimensional reduction and an alternate derivation of string tension better explaining the TBS view of dynamic dimensionality beyond the Dirac hypertube MOU [2,6,21- 
23]. Sufficing for now, reverting to the original form of variable string tension, $T_{S}=\hbar+\Delta T_{0}$ (abandoned because it contained a tachyon deemed unphysical) supports the cyclical KK-like TBS basis of XD space built with an inherent tier of Cramer-like standing-wave components [21]. If nature is stringy, $h$ is not a fundamental constant at the semi-quantum limit; natural units for a string/brane won't have $h=1$, but $T_{S}=1 / \pi$. String tension, $h$ and $c$ combine to form a length, $L$. This means that $h$ in string theory must be multiplied by $T_{S}$. Adapting string theory to XD-LSXD duality suggests $L$ can cyclically oscillate asymptotically to the size of the Lamour radius of the Hydrogen atom - indicia of a gateway to LSXD.

Such ruminations allowed a derivation of a unique M-theoretic vacuum that included a simple preliminary equation related to the dynamics of topological charge phase transitions (governing dynamics of Dirac hypertube), motivating an exploratory inroad into a putative basis for initial superficial parameters of an Einsteinian UFM [23-26] to access XD-LSXD discussed below.
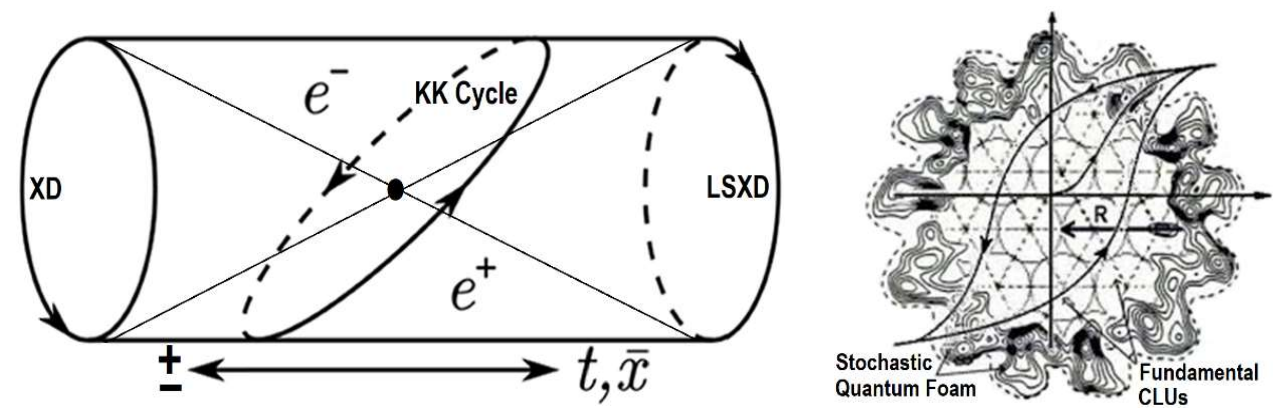

Figure 3. a) Dirac spacetime electron hypertube manifold model. b) End view with polarized vacuum tessellated with CLUs with a hysteresis loop suggesting an applied resonance oscillator may open or close the hypertube.

Most accept by quantum theory and measurement, that if XD exist, since they are invisible, they must be curled up at the Planck scale. This is not the only interpretation. By subtractive interferometry between our 3-brane cycle in time, rotating on a KK-like nonlocal EPR atemporal instantaneity of the dual XD-LSXD M-theoretic manifold of the brane bouquet bulk, the simplified nilpotent WheelerDewitt wave function of the observer, $\hat{H}|\Psi\rangle=0 \quad[27,28]$ is cyclically annihilated-recreated hiding behind the domain wall of the uncertainty principle that the range of collapse extends possibly from the Larmor radius of the hydrogen atom to a virtual $\hbar$ asymptote never actually reached. Although highly speculative, in simplistic defense, the Dirac electron hypertube model suggests it might be correct $[4,5]$. Compare Fig. $2 \mathrm{c}$ to Fig. 3 noting $T_{S}=\lambda+\Delta T_{0}$ with KK cyclicality provides indicia for Dirac hypertube coincidence with the extended electromagnetics of a Dirac polarized vacuum $[4,5]$.

We suggest that Chantler's experiments are indicia of this situation. We will elaborate below in terms of the dimensional conundrum. More saliently the Chantler and putative CERN experiments can at best only produce subtle indicia of QED violation or XD/LSXD respectively because of the 4D limit of the SM and the observational limit inherent in the uncertainty principle. In contrast, our proposed experiment, if successful, promises unfettered low energy complete access to a $3^{\text {rd }}$ UFM regime of reality [2-7].

\section{Challenge of Dimensionality - Only Resolved Experimentally}

"In three dimensions there is not enough room to put all the laws of physics." - Michio Kaku

Clearly Yang-Mills (YM) Kaluza-Klein (KK) correspondence (equivalence) could drive the future of particle physics providing an empirical path extending the SM. Although generally known that YM-KK theories define equivalence on principle fiber bundles; specific conditions for equating their Lagrangian have not been rigorously specified. Since the origin of KK theory, virtually all corresponding extensions of the SM rely on a profusion of additional dimensionality (XD) $[29,30]$; a conundrum that obviously can only be resolved experimentally. For example, Riemannian KK 
manifolds, $\mathrm{M}$ with horizontal and vertical subspaces in the tangent bundle $(\mathrm{M}=\mathrm{X} \times \mathrm{G})$ defined by YM connections are orthogonal with respect to the KK metric, where $X$ is a $4 \mathrm{D}$ spacetime and $\mathrm{G}$ an arbitrary gauge Lie group; and for the corresponding YM theory, $\mathrm{M}$ is a trivial principle G-bundle $[29,30]$. This suggests putative orthogonal extensions of dimensionality beyond the $4 \mathrm{D}$ utilized by the SM requiring a fundamental change in the meaning of the concept of dimensionality [22].

Two special processes emerge for modelling XD: 1) Duality, where the dimensions are fundamentally different in character, and 2) Anti-commutativity, where they are fundamentally the same [22]. The unique complex quaternionic Clifford algebra revealing how to operate the experimental design is under development [34]. Rather than the current iteration of String/M-Theory this work is based on a radical extension of the original hadronic form of the theory because of corresponding key elements such as virtual tachyon/tardon interactions (allowing more than one temporal dimension [35,36]) and a variable concept of string/brane tension, Ts yielding experimental design parameters for accessing additional dimensionality [31,33].

In contrast to ongoing QED violation and CERN LHC SUSY XD experiments, this work explores a radical new M-theoretic / Unified Field Mechanical (UFM) inroad approach surmounting quantum uncertainty outlined in preliminary form [31-33]; which if successful could have far reaching consequences for validating M-Theory and provide tabletop-low energy UFM cross section alternatives for viewing putative SUSY partners (or alternative brane topologies) in a transdimensional slice rather than the $\mathrm{TeV}, \mathrm{PeV}$ supercollider techniques utilized to produce standard cross section particle sprays in the highly successful 100 year history of high energy collision physics.

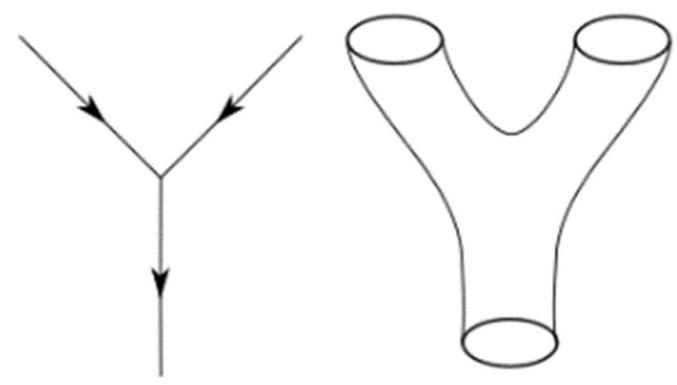

Figure 4. Quantum interactions: a) Standard Model 0D Fermionic point particle world line. b) M-Theory world sheet with 1D string; extended to $M_{12}=M_{4} \times C_{8}$ brane topology in our model where $\mathbb{C}_{8}= \pm \mathbb{C}_{4}$ as a form of $\mathrm{K}-\mathrm{K}$ mirror-symmetric cyclicality.

The KK formalism appeared as the first suggestion of the utility of $\mathrm{XD}$ as a tool in unification procedures. It is generally known that KK modeling makes correspondence to the SM through YM Gauge Theory [29,36,37]. Decades later the concept of Higher Dimensionality (HD) became associated with String Theory; now extended to an 11D M-Theory with Calabi-Yau mirror symmetric brane topology [38]. M-Theory has been severely criticized until now by the inability to perform experimental tests [39].

A salient feature of YM-KK correspondence as a path for extending the SM is the utility of the additional degrees of freedom allowed by dimensionality beyond the $4 \mathrm{D}$ of the SM. That a mathematical YM-KK correspondence exists is reasonably obvious [29,36-43] and not under overt dispute; what is questioned is whether or not extended real physical correlations exist. The debate has continued for at least ninety years. For a modicum of completeness, we initially list a couple formulations briefly here:

A correspondence path to unified theory began in 1919, but not until the 1940's was KK theory completed. Kaluza's 1921 invariant 5D line element is $d s^{2} \equiv \tilde{g}_{a b} d x^{a} d x^{b}=g_{\mu \nu} d x^{\mu} d x^{v}+$ $\phi^{2}\left(A_{\nu} d x^{v}+d x^{5}\right)^{2}$ where $\tilde{g}_{a b}$ is the 5D metric and $g_{\mu \nu}$ the $4 \mathrm{D}$ spacetime metric; $\phi$ is the associated scalar field at a $5^{\text {th }}$ diagonal, and $A$ the Electromagnetic (em) vector potential from which the equations of both General Relativity (GR) and em can be derived [44,45]. 

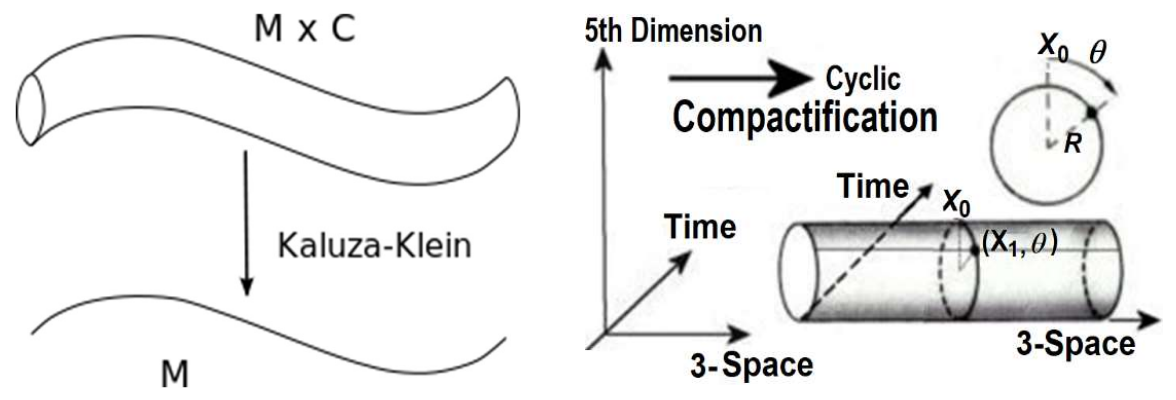

Figure 5. a) KK space, $M \times C$ is compactified over set $C$; KK decomposition produces a field theory over $M$. The tangent bundle of $\mathrm{M}(\mathrm{M}=\mathrm{X} \times \mathrm{G})$ defined by the $\mathrm{YM}$ connection is orthogonal with respect to the KK metric. b) The inherent cyclicality of KK compactification from 5D.

In figure $5 \mathrm{~b}$ Klein suggests $5 \mathrm{D}$ KK-space is closed in the ${ }^{X_{0}}$ direction with a period $\ell$. If QM is applied to the geodesic, $P_{0}= \pm N_{\ell}^{h}$, with $N$ a positive or negative quantum number depending on the direction of the 5D motion. Thus the period, $\ell=h c \sqrt{2 \kappa} / \varepsilon=.08 \times 10^{-30} \mathrm{~cm}$, with $\kappa$ the Einstein gravitational constant and $\varepsilon$ electric charge [46]. We have already claimed KK or M-theoretic XD appear Planck-scale because of the mechanism of the uncertainty principle.

It is possible to have supersymmetry in alternate dimensions because spinor properties change dramatically with dimensionality. For example, in d dimensions, spinor size is $\sim 2^{\mathrm{d} / 2}$ or $2^{(\mathrm{d}-1) / 2}$. The maximum supersymmetry is said to be 32; thus, the greatest number of dimensions in which a supersymmetric theory can exist is $11 \mathrm{D}$. An $\mathrm{SU}(3) \times \mathrm{SU}(2) \times \mathrm{U}(1)$ gauge symmetry group can describe all known particle interactions. Following Witten, [42,43] the minimum number of dimensions of a manifold with this symmetry is 7D. Gauge fields arise in $\mathrm{SU}(3) \times \mathrm{SU}(2) \times \mathrm{U}(1)$ group symmetry in a gravitational field as components of more than $4 \mathrm{D}$. This forms a reality of at least four non-compact and seven compact spacetime dimensions, $M^{4} \times S^{7}=11 \mathrm{D}$, which Witten [43] calls a 'remarkable numerical coincidence' because this 11D supergravity maximum is the minimum for $\mathrm{SU}(3) \times \mathrm{SU}(2) \times \mathrm{U}(1)$ symmetry which for symmetry reasons observed in nature is in practicality the largest group one could obtain from KK theories in seven XD.
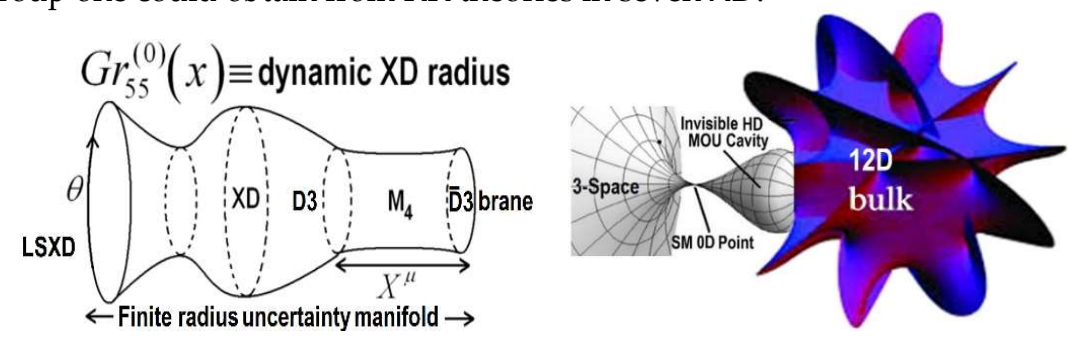

Figure 6. a) Randall-Sundrum warped throat model of dynamic GR radius for LSXD fluctuations, where $X^{\mu}$ are the Lorentz coordinates. Redrawn from [47]. b) Additional XD throat model.

Following Sundrum [47] for 5D GR the Einstein action is $\ni \partial_{\mu}$ or $\partial_{5} G r_{M N}^{0}(x) \rightarrow 0$ for XD fluctuations $d s^{2}{ }_{\ni} G r_{55}\left(d x^{5}\right)^{2}=G r_{55} R^{2} d \theta^{2} \Rightarrow G r_{55}^{(0)}(x) \equiv$ dynamical XD radius. Randall and Sundrum [48] found an HD method for solving the hierarchy problem utilizing 3-branes with opposite tensions, $\pm \sigma$ residing at the orbifold fixed points which together with a finely tuned cosmological constant form sources for $5 \mathrm{D}$ gravity.

The various Randall-Sundrum models utilize a 5D warped geometry to describe reality as an anti-de Sitter (AdS5) space with elementary particles residing on a localized $3+14 \mathrm{D}$ brane (D3 Planck brane) and an additional separated gravity brane. The Randall-Sundrum warped AdS ${ }^{5} \mathrm{XD}$ postulate 
aligns sufficiently with our finite radius manifold of uncertainty (MOU) [30,49] to give a semblance of credibility to each. Technological access to XD-LSXD requires a new group of transformations beyond the Galilean-Lorentz-Poincairé.

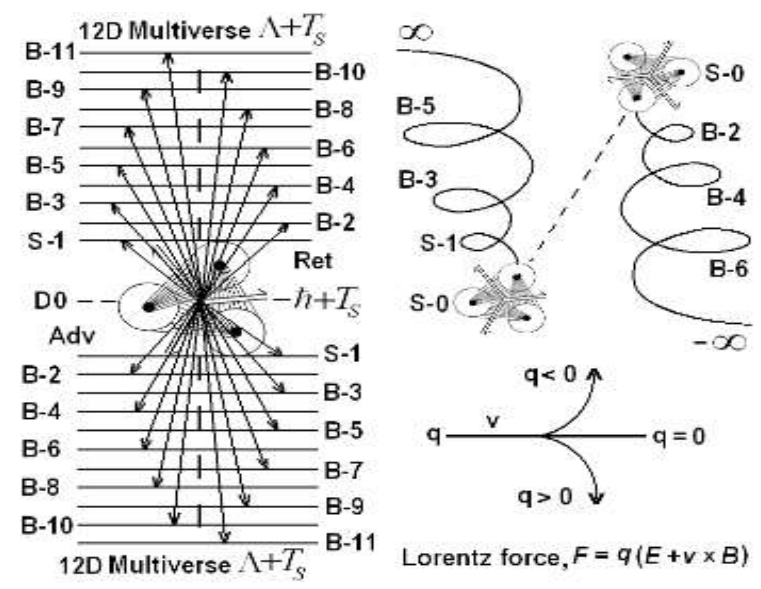

Figure 7. Conceptualized string (S) and brane (B) couplings in advanced-retarded mirror symmetric Calabi-Yau spacetime arising from Cellular Least-Unit (CLU) translations. a) String-brane duality couplings from 0 to $12 \mathrm{D}$ for odd-even HD brane topologies. b) Ising model spin-glass rotations which may be driven by an internal Lorentz-like UFM force of coherence or applied external resonances for vacuum engineering technologies.

Every Calabi-Yau manifold with mirror symmetry or T-duality admits a hierarchical family of supersymmetric toroidal 3-cycles. It is currently undefined theoretically whether the attempt to formalize this compactification-boost continuous-state structure should follow a KK spin tower, logarithmic or golden ratio spiral, cyclotron resonance hierarchy, genus-1 helicoid parking-garage or some other HD topological structure [2]. We currently find the Genus-1 helicoid logically appealing because of its ability to incorporate Kahler manifolds compatible with M-Theory parameters. Also of note is that the heterotic $\mathrm{SO}(32)$ Bosonic string introduces a tachyon which we do not consider anomalous but part of the internal field coupling of a Lorentz vacuum contraction [33]. Type IIA \& Type-IIB open/closed strings are cast in odd/even string/brane dimensionality which we postulate is an inherent part of the Ising model rotation of the Riemann sphere for 'genus-1 parking-garage helicoid' raising-lowering indices of the continuous-state dimensional reduction compactification process [2]. These complex UFM constructs can only be adequately worked out with a move away from limits imposed by Copenhagen-Gauge approximations.

Because of the numerous unresolved attempts at finding rigorous realistic XD model building one is justified in claiming the issue can only be resolved by experiment. Four putative empirical search avenues for additional dimensions (XD) are concurrently being explored:

1. CERN - LHC: Search for LSXD predicts a 'leaking' of gravity between HD branes as in the RandallSundrum model $[47,48]$ where the visible, $4 \mathrm{D}$ universe is restricted to a brane inside an HD space called the bulk (Fig. 8). If true one claim is to be suggestive of mini black holes (MBH), the energy of which is calculated in terms of what theorists' call 'gravity's rainbow' [50-53]. The detection of $\mathrm{MBH}$ suggests existence of LSXD. 5.3 TeV tests were unsuccessful. Analysis predict that black hole detection requires a minimum of $9.5 \mathrm{TeV}$ for $6 \mathrm{XD}$ and $11.9 \mathrm{TeV}$ for $10 \mathrm{XD}$. Absence of results so far is suggested as an "indication of a suppression of higher dimensional black hole production due to Planckian deformation of quantum gravity which was not taken into account" [40]. 'Using gravity's rainbow, it was found that the energy needed to form $\mathrm{MBH}$ is larger than the energy scale of the LHC, but said to be within reach of next generation particle colliders' [51-53]. 


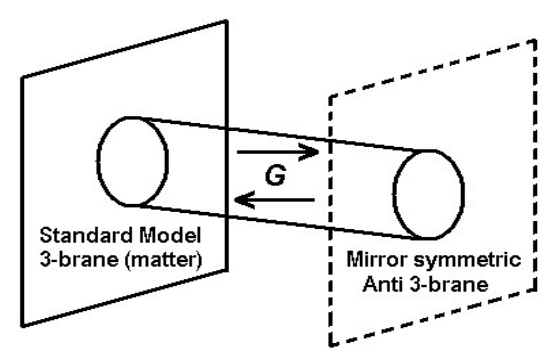

Figure 8. D-brane model of $4 \mathrm{D}$ SM on a 3-brane with $G$ in $10 \mathrm{D}$ able to pass through the HD bulk by mirror symmetric brane-antibrane topological phase interactions.

2. Synthetic dimensions in quantum Hall graphene bilayers. Synthetic dimensions produced by topological phase transitions in quantum Hall bilayers could lead to the discovery of actual XD if topological protection is overcome and topological phase transitions are understood beyond 2D. $[54,55]$.

3. Testing the Casimir force in different isotopes of the same element. Recent Casimir related XD theories suggest the possibility of new short-range gravitational forces. The prediction is that new Casimir forces at sub-micron separations between test bodies composed of different isotopes of the same element may arise from new spatial XD revealed in the iso-electronic effect of different isotopes [56-58].

4) Tight-Bound States (TBS) in the hydrogen atom. Detection of additional spectral lines in hydrogen below the lowest Bohr orbit in hyperspherical XD cavities at the semi-quantum limit utilizing an incursive oscillator beat frequency resonance [10-12].

Since quantum mechanics cannot be considered the so-called basement of reality, Yang-Mills Kaluza-Klein equivalence is provided as an empirical path extending the standard model of particle physics to include supersymmetry [30]. Feynman proposed a synchronization backbone; we implement this concept by eliciting a novel approach to the Wheeler-Feynman-Cramer Transactional Interpretation of quantum theory where a present instant is a hyperspherical standing-wave of the future-past. This relies on a Dirac covariant polarized vacuum with an inherent beat frequency in the spacetime backcloth based on Feynman's concept of a synchronization backbone [59,60]; utility of which allows the uncertainty principal to be supervened cyclically by an rf-pulsed incursive resonance oscillator. Simplistically, the protocol is a hierarchy consisting of electron-nucleonspacetime spin-spin coupling resonance to induce destructive interference within an oscillating radius determined by incursive oscillator parameters. Reliance is made by suppositions of the Dirac electron hypertube, Randall-Sundrum warped D-brane wormhole and Bohm's superimplicate order. The key element is extension of the Vigier model of Tight-Bound States (TBS) in the hydrogen atom. The experimental result is the detection of 2 or 3 additional spectral lines in hydrogen below the lowest Bohr orbit (TBS) which can be precisely predicted utilizing the 1895 equation for the volume of hyperspherical cavities for new lines in 4D, 5D and 6D XD.

Atomic physics conventionally treats electromagnetic interactions other than Coulomb spinorbit or spin-spin couplings as perturbations with small corrections to the energy levels. Coulomb's electrostatic inverse square law is, $\left|F_{C}\right|=k_{e}\left(\left|q_{1}, q_{2}\right| / r^{2}\right)$, where $k_{e}$ is the Coulomb constant and $r$ is the Bohr radius. Seminal work by Vigier and colleagues proposed the creation of strong magnetic interactions at small distances and the creation of anti-Born-Oppenheimer states corresponding to rapid motion of the heavy particles around essentially fixed electrons. These four models are prime candidates for empirical tests of physics beyond the SM.

\section{What is Matter? - An Evolutionary Step}

Matter is primarily known as observable extension, duration and particle field interactions occupying the spacetime continuum; which by QED we know the relative distance between the 
proton and electron in a hydrogen atom to be $99.999 \%$ empty space. The imminent paradigm shift toward Unified Field Mechanics (UFM) entails passing beyond observation relative to the spacetime continuum into a spatial regime of XD-LSXD duality where matter takes on additional topological properties entailing continuous-state Calabi-Yau brane bouquet phase transformations. Therefore, an essential broad-based extended model must be introduced, with cursory correspondence to current thinking in string / M-theory which seeks a single unique XD compactification to SM spacetime. In line with KK cyclicality, we introduce a periodic continuous-state compactification process (Fig. 7); essential because the local-temporal $3(4 \mathrm{D})+++-$ observer subspace manifold, must make correspondence to nonlocal-instantaneous XD-LSXD M-theoretic/UFM topological phase space $[6,24]$. Heisenberg potentia (before measurement) is suggested to be infinite by Copenhagen QM. This conforms with spacetime of the observer being nilpotent. A quantum mechanical nilpotent field allows accessibility to coherent elements of UFM (coherent control of HD topological phase transitions) allowing pragmatic protocols for surmounting the barrier of the uncertainty principle providing utility for to accessing the duality of XD-LSXD space. Figure 9 diagrams M-theoretic dualities of the brane bulk bouquet as interpreted by Huerta [61].

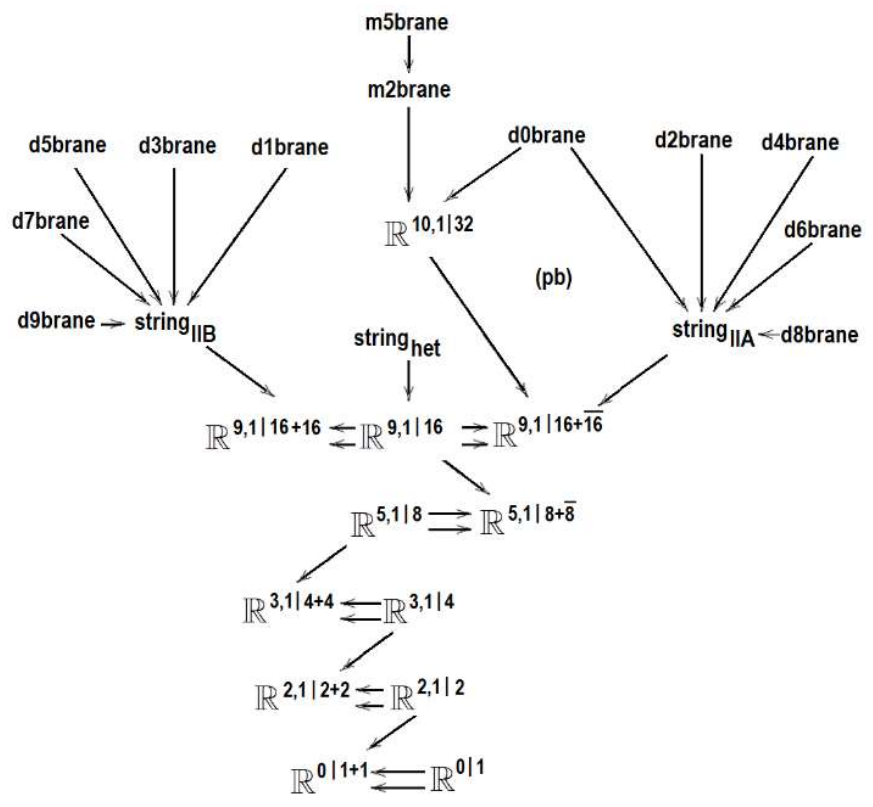

Figure 9. 11D M-theoretic version of the brane bouquet unifying the 5 string theories, Fig. adapted from Huerta [61].

In addition, we must clarify that XD M-space is not a SM submanifold; spacetime of the observer is the submanifold. For centuries natural science suffered from geocentric cosmology; similarly, now the Terran observer considers itself the center of intelligence rather than a local distinction in a holographic anthropic multiverse [2]. Logic for this insight is simple - An Einstein brane bouquet unified field is noted as the mother of all fields - not the case for classical - quantum mechanical SM limits of virtual observables. CERN clerics claim discovery of XD demonstrates the existence of a multiverse - infinite number of nested Hubble spheres beyond the observational limit - each by a finely-tuned Terran fine structure constant to radii of $\sim 13.7$ billion light years [3]. Note that while beyond the scope of this paper, as surmised from Fig. 8, dark matter/energy simply signifies the gravitational presence of the multiverse.

Because the spacetime KK-like cycle at the XD manifold entails an inherent beat frequency (Sect. 5), resonant interference can be achieved because of the nature of the Dirac polarized covariant vacuum and the inherent Dirac hypertube array within it [4,5]. Figure 9 illustrates a first indicia for conveying this continuous-state cyclic process. Let the two Bloch spheres represent resultant points of an SM line element. Galilean-Lorentz-Poincairé line elements transforming with infinitesimal distance between points contain only usual SM information. With the addition of a KK-like cyclical 
XD brane topology on the top row of Fig. 10 (represented by a space-antispace mirror symmetric quaternion algebra) topological phase transitions undergo a continuous-state periodic $\mathrm{XD}$ reduction to SM 3-space. This process is closed to observation by the domain wall of the uncertainty principle.

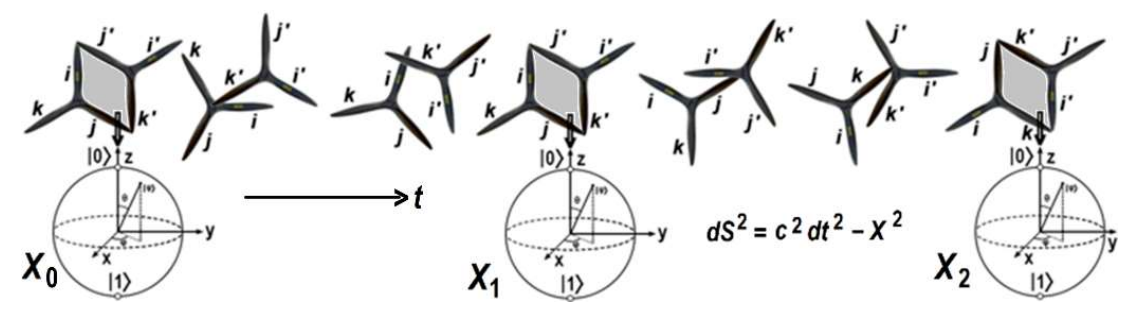

Figure 10. Locus of nonlocal HD space-antispace mirror symmetric Calabi-Yau 3-tori spinning relativistically and evolving in time. a) Top row. Topological phase (depicted by quaternions) of an XD cycle progressing from chaotic to periodic nodes coupling, compactifying by dimensional reduction into resultant (cube faces) 3-space quantum states depicted in b) Bottom row, as Riemann Bloch spheres, indicative of the emergence of observed 3D reality from the XD-LSXD duality brane bulk.

Figure 10 (bottom) illustrates an SM line element, locus of semi-classical Riemann Bloch 2spheres, $X_{0}, X_{1}, X_{2}$ as basement of reality. At the top, $1^{\text {st }}$ space-antispace mirror symmetric UFM step of quaternionic vertices cycling from 5D QM chaos to topological order as faces of 3-cube. We also see the beginning of separation of the standard 3-space line element into an extended KK cyclicality from order to chaos at the semi-quantum limit. The inherent beat frequency is revealed by rfmodulation of the Dirac polarized vacuum. When the stochastic background coheres into the square.

String theory essentially has a single parameter - string tension, $T_{S}$ which is modulated by the string coupling constant (ill determined at present). The lore states simply, all matter is comprised of vibrating strings. M-theory also currently remains silent in any attempt to configure a bouquet of resonant strings into a fermion (see Sect. 7). The googolplex of possible Calabi-Yau configurations, $10^{\infty}$ for deriving a single unique compactification of the 12D bulk producing the $4 \mathrm{D} S \mathrm{SM}$ has remained elusive. While we have been able to derive a unique vacuum utilizing an alternative derivation of string tension in a continuous-state cosmology [2,3,23], our adapted anthropic M-theoretic/UFM model, may provide indicia of feasibility of such modeling on the horizon; success would be elusive without a new set of UFM group of transformations beyond the Galilean-Lorentz-Poincairé. At this point in development we are able to add one significant parameter that turns out to be an essential element in the TBS protocol design (Sect. 5).

All atomic matter appears to 3-space observation as a singularity. In wave-particle duality, the quantum field is like clothing dressing a OD particle. Physical science has no idea what the fundamental nature of a field is; we put a metric in its proximity and measure salient properties. Likewise, we do not know what space is, other than to apply Einstein's definition, that it is extension. Our observations of matter in 4D Euclidean-Minkowski-Riemann space are considered geometric. The additional $8 \mathrm{D}$ of the M-theoretic UFM bulk are considered topological, with attempts underway to provide topological field theories $[5,24,25,49]$.

Wave-particle duality, is a probabilistic Heisenberg potentia given either wave or particle parameters depending on measurement conditions. This QM regime is only an oasis in the combinatorial hierarchy of reality $[2,5,24,25,62-64]$. However, in passing beyond the Copenhagen wall of QM exclusion/uncertainty, the inherent processes of existence are a continuous cycle of this duality and its extended mirror symmetric or supersymmetric partners as to be determined.

As well-known, Einstein claimed, if one put a saddle on a photon, one could circumnavigate the universe without the passage of time or looking into space far enough, ones sees the back of one's head. The latter (known as the wrap-around universe) has not been excluded by Planck satellite data [65-67]. This is not all; its observation pertains to the nonlocal instantaneity where temporal dimensions are transformed by annihilation. 

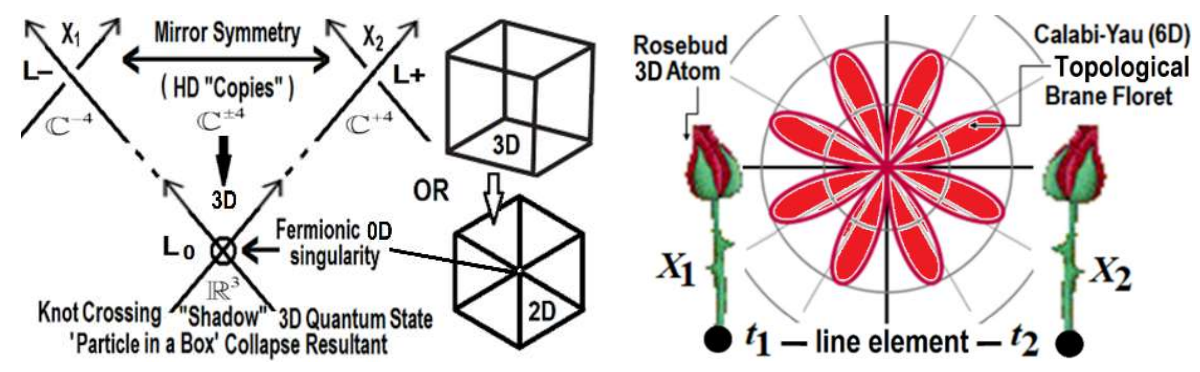

Figure 11. XD-LSXD UFM models of matter. a) An oriented left-right (over-strand, under-strand) crossover link diagram; each component has a preferred direction as shown by the arrow. For a given crossing $L_{+}, L_{-}$, resultants $L_{0}$ and $\mathbb{R}^{3}$ change the diagram as proposed. Braid elements in the HD complex, $\mathbb{C}^{ \pm 4}$ brane world become a knotted shadow when projected onto Euclidean space, $\mathbb{R}^{3}$. For a space-antispace configuration, $X$ would only represent the knot shadow, fermionic singularity of observation in b) with Euclidean coordinates $x, y, z$. b) Illustrating how a crossing shadow reduces dimensionality. c) Consider $X_{1}\left(t_{1}\right)$ and $X_{2}\left(t_{2}\right)$ as ends of a line element between two atoms depicted in 3-space as rosebuds.

The bottom cross of Fig. 11a shows a 3-space knot shadow fermionic singularity, $L_{0}$ by the collapse of XD topological mirror symmetry. The localized knot shadow hides XD behind the uncertainty principle. Figure 11c is simplistic in that the rosebud is only illustrated for an $x$ coordinate; whereas it is proposed that a quaternionic space-antispace mirror symmetric representation, $\pm i, j, k$ would entail six buds continuously blooming (Calabi-Yau brane topology) and compactifying into 3-space knotted shadows. A knot projected onto a plane casts a shadow. Small changes in the angle of projection shows if it is one-to-one except at the crossings, where a shadow of the knot crosses itself once transversely. Analogously, knotted surfaces in 4-space relate to immersed surfaces in 3-space.

The knot crossing shadows in Fig, 11b in the next XD step are illustrated as trefoil knots. In work in progress, we show that the topology of the Dirac spinor is a trefoil in HD; a fact hidden from observation, until now, by the uncertainty principle.

\section{EXTENDED VIEW OF REALITY}

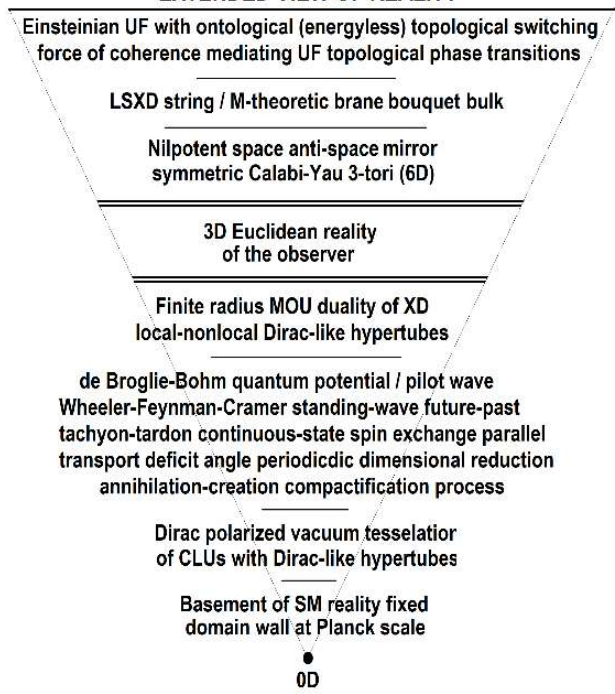

Figure 12. A new extended view of reality showing structural phenomenology at various levels of the hierarchy.

In this section, we attempt to provide insight into the dynamics of brane topological in the bulk. Radically divergent from string communities current thinking, primarily because compactification is a cyclic continuous-state. We will not argue this point now, other than proposing in passing, that the reason is anthropic. Einstein himself stated that his long sought UF could explain living systems.

Fermionic matter can no longer be considered 0D point particles (electrons) or as rigid Mass-charge 
quark microspheres (nucleons), as treated by vector algebra or quantum field theory, embedded in (3)4D (+,+,+,-) Minkowski-Riemann manifold; but must now be devised as a compactification of 6D D-brane mirror symmetric Calabi-Yau florets cyclically driven by de Broglie-Bohm-Cramer piloted matter-wave XD brane topology-phase transitions. The modified model of matter, albeit incorporating relevant off-the-shelf, parameters currently incorporated into the vast panoply of thinking comprising String / M-theoretic parameters; especially those related to T-Duality D-brane mirror symmetry, because as generally known, T-duality interrelates two theories with different spacetime geometries. Thus, allowing correspondence with usual notions of classical atomic geometry, quantum field theory or our radically different UFM formulation of T-duality.

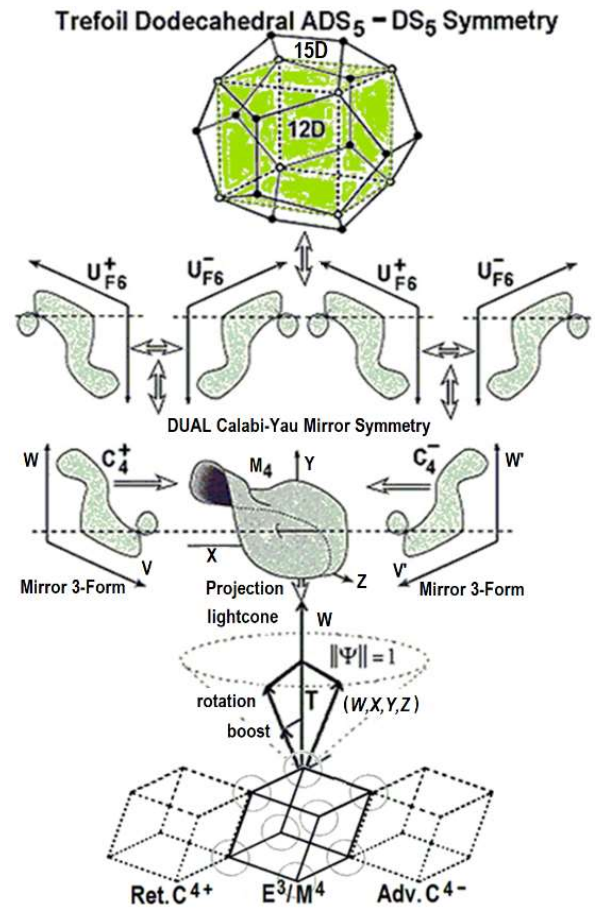

Figure 13. Axiomatic completion of Fig. 10 adding 8D to 12D (embedded 15D) illustrating full extended rendition of additional parameters for a relativistic XD quantum state and instantaneous LSXD in continuous-state dual Calabi-Yau mirror symmetric UFM cosmology representation that has sufficient parameters to surmount the uncertainty principle. a) Bottom row. Replacing the Bloch 2-sphere representation (Fig. 10) with a Cramer advanced-retarded future-past 3-cube resultant.
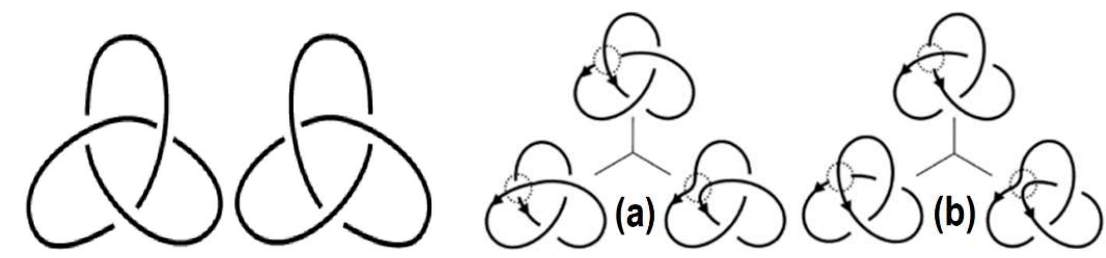

Figure 14. a) Left and right-handed trefoil knots are mirror images of each other. b) Raising and lowering of trefoil over and under crossings allows a variety of topological moves likely pertinent to brane matter.

Topological moves (phase transitions) are richly endowed. We are attempting to illustrate the battery of parameters useful in describing the operation of the UFM transformation group. We will continue to briefly address these elements in an introductory manner in sections that follow primarily to give an overview of the requirements as seen at this point in time in the hopes of engendering interest in further development to address vacuum accessibility. 

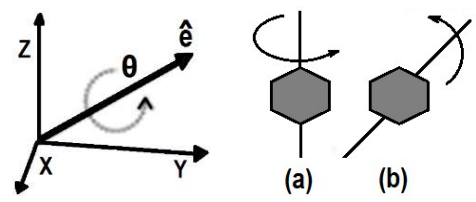

(a)

(b)

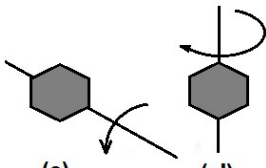

(c)

$\left(a^{\prime}\right)$

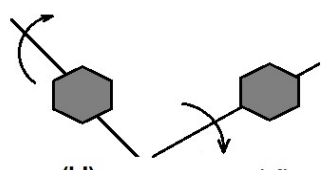

(b')

(c')

Figure 15. In regards to Fig, 14a, unknotting the crossover links during parallel transport spin exchange deficit angle dimensional reduction conditions allows rotations to be added to topological phase transitions.

The local SM component corresponds to 4D quantum Field Theory wherein Copenhagen aspects are replaced by resultant Cramer Transactional and extended de Broglie-Bohm piloted implicate order causality with a corresponding nonlocal duality of Large-Scale Additional Dimensionality (LSXD) in the bulk described by an Ontological-Phase Topological Field Theory developed as a 12D form of cyclic Kaluza-Klein theory initially introduced by Yang-Mills Kaluza-Klein correspondence to include a fundamental Cellular Least Unit (CLU), the primary requirement for an Einsteinian Unified Field Theory as the tessellation of space/spacetime [29-33,68].
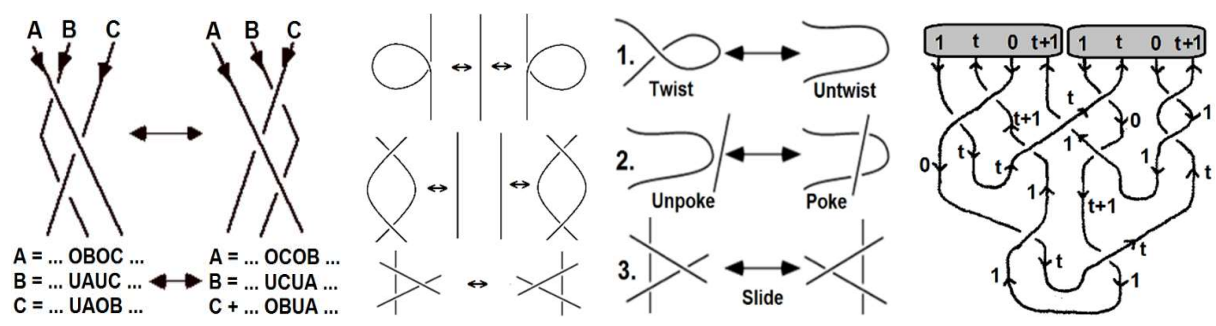

Figure 16. $a, b, c)$ Varieties of Reidemeister moves useful to study as likely apply to parallel transport in the continuous-state dimensional reduction process in the XD-LSXD duality cycle. d) A spacetime roll spun knot.

To fully represent matter up to and beyond space-antispace, three oriented rosebuds $X, Y, Z$ would be required mirror symmetrically designated with quaternion notation, $Y=i, j, k$ and $Z=-i,-j,-k$. In addition, the space-antispace configuration would be represented as in the center of a) as rose petals in bloom. $X, Y, Z$ undergo Dirac spinor rotation, where a rotation through antispace takes $720^{\circ}$ rather than the $360^{\circ}$ needed for a 3-space rotation to return to the starting position. Because this only represents the penultimate compactification to 3 -space, $X, Y, Z$ are represented by a trefoil configuration of three sets of quaternions. This fact has been hidden from observation as an element of the Dirac spherical rotation by the quantum uncertainty principle. To complete the UFM line element cycle the rose petal symmetry (Calabi-Yau topological brane interactions), continues through a continuous 12D KK-cycle. The mirror image of the space-antispace mirror image is causally free of the knot crossing shadow in 16b), which is realized or the collapsed resultant of the local 3(4)D Euclidean/Minkowski quantum particle-in-a-box.
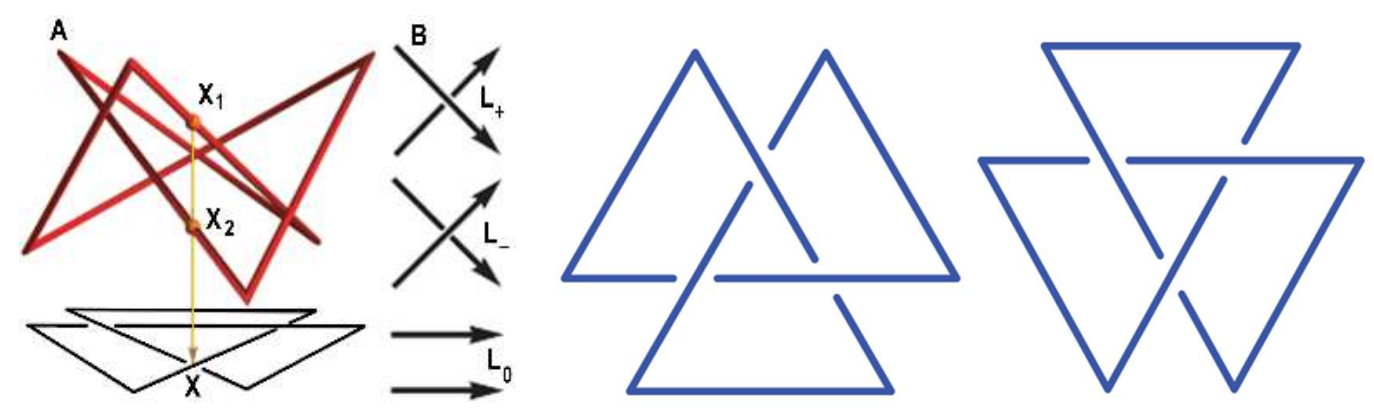

Figure 17. a) Knot diagram of the Conway skein triple with the 2D shadow representing topological reduction form XD space, and b) crossover links. Adapted from [69]. c,d) Visualization of L-R trefoils in triangular form for ease of visualizing relation of overlap to hexagonal CLU geometry Dirac vacuum micromagnetics. 
In Fig. 17a A) is a 3D polygonal representation of the trefoil knot (red) and its planar diagram (black). Two red spheres on the knot mark the 3D points $X_{1}$ and $X_{2}$ projecting down to $\mathrm{X}$ on the planar diagram along the brown arrow. B) The Conway skein triple is composed of three oriented diagrams that are the same outside a small region, where they look like the illustrated $L_{+}, L_{-}, L_{0}$. To define the oriented sign of a crossing, approach it along the underpass in the direction of the orientation: if the overpass orientation runs from $L$ to $R$, the oriented sign is $+1,-1$ otherwise [69].

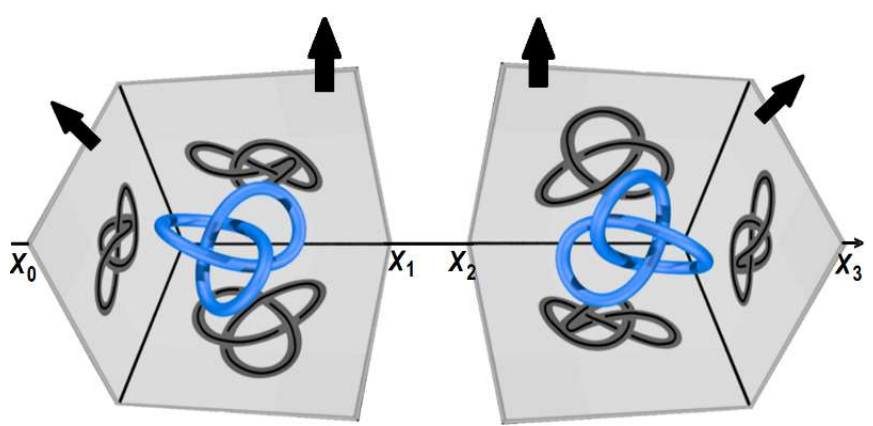

Figure 18. 3 Projections of trefoil knot; black arrow point toward XD-LSXD space with X coordinates representing a Euclidean line element with the trefoils meant to be HD dynamics hidden by the uncertainty principle in the infinitesimal distance between temporal points. Adapted from [70].

It is commonly believed that the Dirac $360^{\circ}-720^{\circ}$ spinor rotation can be considered as a Klein bottle comprised of two Mobius strips. Our proposal that Dirac spherical (spinor) rotation $\left(360^{\circ}-720^{\circ}\right)$ for SM particle physics in Minkowski/Riemann space 3(4)D +,+,+,- includes hidden trefoil crossover topology in the semi-quantum interface requires further discussion on the parametrization of the trefoil surface in relation to the Dirac Mobius. Because of the existence of HDLSXD duality, hidden (until now) from observation by the uncertainty principle, takes the form of a mirror symmetric trefoil with utility of its over/under crossings for parallel transport in both simply and multiply-connected topologies. This scenario is required to describe the UFM transform and operation of HD M-theoretic bulk reality.

Firstly, the Trefoil, $T$ has polar equation $r^{3}=2 A \cos (3 \theta)$ and Cartesian coordinates $\left(x^{2}+y^{2}\right)^{3}=A\left(2 x^{3}-6 x y^{2}\right)$. Following Langer \& Singer's [71] description of the trefoil as a sextic curve with exceptional properties, such as a genus-1 Platonic surface with 18 equilateral triangle faces that may be exchanged and rotated like the faces of an icosahedron (dual of dodecahedron).

Dixon elliptic functions, based on the curves $x^{3}+y^{3}-3 a x y=1$ and the trefoil make a perfect fit. Our interest is in the Fermat cubic, $x^{3}+y^{3}=1$, for which when $a=0$, the Dixon functions display a unique hexagonal symmetry; a simpler curve with the same projective symmetry group as $T$. Especially as the Dixon sine $\operatorname{sm} z$ can be used to map a regular hexagon onto a Riemann sphere, where the hexagon interior is conformally mapped onto the complement of the three rays joining $\infty$ to a cube root of unity. In this sense, arc length parameterization provides the trefoil's structure as a genus-one Platonic surface, whose 18 equilateral-triangular faces may be arbitrarily exchanged and rotated, like the faces of an icosahedron [71].

The trefoil has many noted features. The Dixon sine equation $\operatorname{sm} z=\tan \frac{p}{2} e^{i \lambda}$ correlates the hexagon point with complex coordinate $z=x+i y$ with the point in the sphere of latitude $\pi / 2-p$ and longitude $\lambda$. The function $w=\operatorname{sm} z$ defines real $z$ by $z=\int_{0}^{w} d x /\left(1-x^{3}\right)^{2 / 3}$, and $\operatorname{cm} z$ by $\mathrm{sm}^{3} z+\mathrm{cm} z=1$. Then interestingly, $\mathrm{sm}(0)=0, \mathrm{~cm}(0)-1$, and $\frac{d}{d z} \mathrm{sm} z=\mathrm{cm}^{2} z, \frac{d}{d z} \mathrm{~cm} z=\mathrm{sm}^{2} z$. In 1896, Caley finally paved the way for formalizing $\operatorname{sm} z$ and $\mathrm{cm} z$ as elliptic functions. 
Since $\operatorname{sm} z$ and $\mathrm{cm} z$ have periods $p_{1}=3 K$ and $p_{2}=3 \omega K$, with $\omega=-1+i \sqrt{3} / 2$ as a cube root of unity: $\operatorname{sm}\left(z+3 \omega^{j} K\right)=\operatorname{sm} z, \operatorname{cm}\left(z+3 \omega^{j} K\right)=\mathrm{cm} z, j=0,1,2$. As for any elliptic functions, one can describe the values of $\mathrm{sm} \mathrm{z}$ and $\mathrm{cm} \mathrm{z}, z \in \mathbb{C}$, via a tiling of the plane by copies of a 'period parallelogram' $P$ with edges corresponding to the pair of periods. There is a corresponding triangulation of $P$ by 18 equilateral triangles. One may also define rotational and quasiperiodic translational symmetries [71].

The tiling of $\mathrm{sm} \mathrm{z}$ and $\mathrm{cm} \mathrm{z}$ trefoil properties to the vacuum are an entry point for applying micromagnetics to the Dirac polarized vacuum. When a static electric dipole $d$ is placed in front of an ideally conducting wall, it interacts with its mirror image. In historical terms, this Casimir-Polder (CP) result, gives the interaction potential between a ground state atom and a mirror as obtained within the CQED (cavity-QED) framework known to be valid for any separation $z$ between the atom and the mirror and results from modification of vacuum fluctuations by the mirror. Recent experiments have given clear evidence for the existence of retardation terms in the atom-wall problem, in good agreement with Casimir-Polder predictions. We extend these parameters in a $3^{\text {rd }}$ regime M-theoretic UFM approach to enable aspects of the Static-Dynamic (S-D) Casimir Effect in relation to topological charge inherent in cyclical (KK-like T-duality) brane interaction dynamics mediated by a super quantum potential of the unified field. The UFM interaction is an Ontological (energyless) transfer of information, not phenomenological (quantal) as in quantum field theory [6].

Returning to the simplistic notion of a nilpotent Wheeler-Dewitt wave function of the universe, $\hat{H}|\Psi\rangle=0 \quad[27,28]$ we would like to save theoretical rigor until later and philosophically discuss what natural science might be like after the imminent paradigm shift to a post M-theoretic Einsteinian unified field theory in terms of a UFM reality wave of the observer. What lurks behind the domain wall of uncertainty? Observation tells us there is more matter in the universe than antimatter; let's provide indicia that they are equal by assuming that the wave structure of UFM reality takes a sine wave form as illustrated simplistically in Fig. 19. If reality is truly holographic, coherent control of the unified field my project a reality wave through a 2-sphere creating a 3D hologram temporally evolving. Rauscher has shown that spatial dimensions may undergo a Lorentz boost transform into a temporal dimension [3]. A $2^{\text {nd }}$ boost could topologically transform the complex temporal boost into a Bohm super-potential de Broglie pilot-wave which in UFM parlance would mediate the unified field force of coherence.

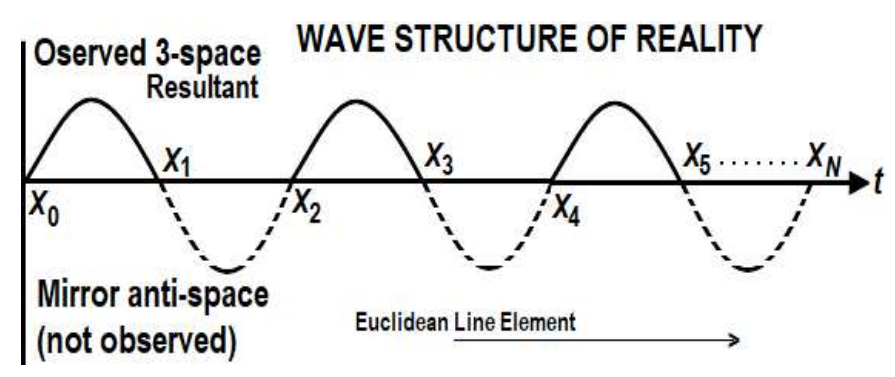

Figure 19. Mirror symmetric wave structure proposing reality is a sine wave evolving in time, where the negative half of the reality wave is annihilated from view by destructive interference of KK-like cyclicality as an inherent aspect of the dynamics of the Uncertainty Principle limiting XD observation of the 3-space observer.

Here loom our indicia; Quigg created a theoretical representation of the underlying structure of SM leptons and quarks he calls the double simplex representation, because the L-handed and Rhanded particles each form a simplex (with antimatter a separate, inverted double simplex) [72]. Two types of quarks make up protons and neutrons inside atomic nuclei; the up quark, which possesses a $2 / 3$ unit of electric charge, and the down quark, with electric charge of $-1 / 3$. Up and down quarks can be either L-handed or R-handed depending on whether they spin clockwise or counterclockwise in respect to their direction of motion. For the Weak Charge, L-handed up and down quarks may 
transform into each other, via weak force interactions when the quarks exchange a $\mathrm{W}$ boson, with electric charge of either +1 or -1 .

In support of Fig. 20, there are no R-handed $\mathrm{W}$ bosons found in nature. This means R-handed up and down quarks cannot emit or absorb W bosons, and don't transform into each other. We have claimed an alternative interpretation of the view that XD must be curled up at the Planck scale because they are invisible. Alternatively, as suggested in Fig. 20, the SM 3-space observer only sees half of the reality wave, mediated by - let's call it a holonomic realiton, which by nature of the unified field would transfer information/energy (topological charge) ontologically (energyless topological phase transition) [6].
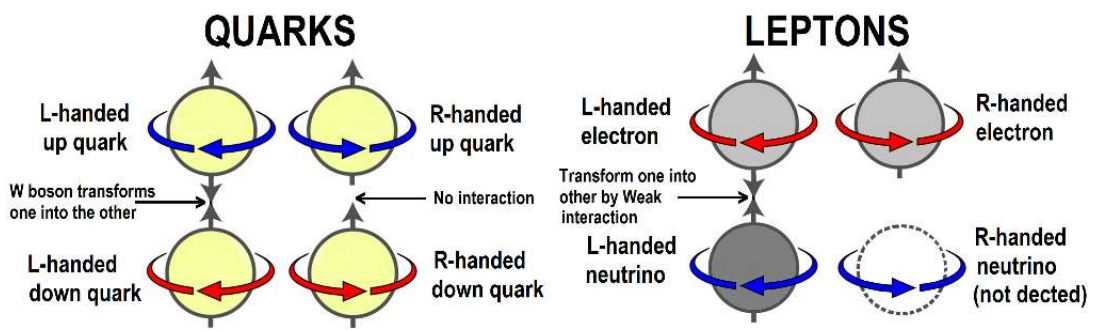

Figure 20. Missing interactions (blocked from observation by Copenhagen Uncertainty Principle) from SM particle physics tools of observation, postulating only half of reality is observed, suggesting reality is like a sinusoidal wave and that in multiverse reality there are equal parts matter and antimatter.

Quarks also possess a Strong Force charge called color with red, green or blue color charges binding quarks of different colors into composite particles such as protons and colorless neutrons with no net color charge. Quarks transform from one color to another by absorbing or emitting gluons, that mediate the strong force. Because gluons possess color charge themselves, they constantly interact with one another as well as with quarks.

Considering the leptons, the other form of matter particles which come in two types: electrons, which have an electric charge of -1 , and neutrinos, which are electrically neutral. As with L-handed up and down quarks, L-handed electrons and neutrinos can transform into each other via the weak interaction. However, again in support of a reality wave, R-handed neutrinos have not yet been observed [72,73].

\section{The Synchronization Backbone of XD-LSXD Periodicity}

Since tests of the uncertainty principle, $\Delta X \cdot \Delta \rho_{X} \geq \hbar ; \Delta E \cdot \Delta t \geq \hbar$ are sacrosanct and myriad, one might pertinently ask why should a, in many respects, straightforward simple experiment be able to surmount the uncertainty principle? Firstly, nature already does this routinely behind the veil of uncertainty. But it becomes scientifically accessible because of the inherent existence of what Feynman proposed a synchronization backbone (originally as a requirement for Quantum Computing [59.60], which is like getting half of the protocol for free. During a standing-wave futurepast Cramer transaction [21] the polarized Dirac vacuum CQED hypertube opens and closes cyclically for a resonant signal to return relatively easily, but to transverse the XD MOU fully to access LSXD is suspected to be much more difficult. A Bessel function incursive harmonic oscillator must be precisely linked/coupled to a resonating mirror symmetric Bessel function with each destructive node precisely linked to the nearby levels of the synchronization backbone hierarchy (Table 1).

The number of nodes has not yet been rigorously determined because supersymmetry/mirror symmetry is not sufficiently understood in terms of topological phase transitions. Ultimately, they will have to be determined experimentally one MOU hyperspherical cavity at a time. We do know theoretically that at 12D the XD-LSXD cycle fully commutes and the final periodic copy of the 3-space CQED particle-in-a-box is causally free. We also suspect by applying conformal scale-invariance, that the MOU structure is not simply connected. This is discerned because cosmology suggests we live in 
a dodecahedral wraparound universe [65-67]. This additionally suggests the need for an ontological phase topological field theory (OPTFT) beyond the confines of quantum locality and unitarity in order to provide the utility for the UFM force of coherence $[6,25,49]$.

Mathemagicians are capable of formulating an equation to describe anything at all, whether it relates to natural science or not; this is the absolute nature of pure mathematics. However, the algebras of Quaternions and Octonions seem to have an inherent ability to describe reality built into them. In that respect, we have found, in preliminary form, a quaternion form that algebraically reproduces the space-antispace face of the periodic resultant cube as depicted in Fig. 10 [80,81]. Our presumption is completion of that program will be an aid in embedding a specific Bessel function with nodes of the Dubois incursive oscillator [82-83].

The nilpotent quaternionic representation of fermions is a powerful method of quantum mechanics and quantum field theory, which also relates to the question of dimensionality, and can be represented algebraically as a double vector algebra, a vector quaternion algebra, or a complexified double quaternion algebra. All three representations are significant, vector quaternions (also includes real complex numbers as subalgebras) arises from a direct combination of the algebras of the four fundamental parameters in physics: space gives us vectors, charge quaternions, time complex numbers, and mass real numbers. The double vector algebra expresses the fact that the whole of physics, and in particular the representation of a point-like fermion, can be seen as a combination, and even a cancellation at a point, of two vector spaces: real space and the antispace constructed out of the combination of charge, time and mass. Complexified double quaternion algebra can be seen as the basis of the representation of a physical object using the parameters space, charge, time and mass as a broken octonion, an octonion in which parts that are antiassociative are also nonphysical. This approach arises from two fundamental propositions. One is that reality represents a fundamental nilpotent zero totality.

This can be achieved through a fundamental symmetry between the parameters mass, time, charge and space. Symmetry is obtained by placing properties against antiproperties, which are exact opposites. Group information about the universe is assumed to be absolutely exclusive; meaning, there is no information about the universe, other than what comes from the parameter group. The properties and antiproperties are absolutely opposite in every respect, though the designation of which are properties (conserved, real and commutative) and which antiproperties (nonconserved, imaginary and anticommutative) is arbitrary. An investigation of the foundations of physics tells us that the properties and antiproperties are exact. Properties and antiproperties can be represented algebraically in a way that makes the zero totality even more explicit. A nilpotent reality supports our supposition that spacetime of the SM observer is cyclically annihilated (closed) and recreated (opened) by a coherent incursive subtractive interferometry of the Unified Field, resulting in a domain of uncertainty as a barrier to observation of XD and M-theoretic brane parameters of matter. Symmetries in the synchronization backbone within the Dirac vacuum facilitate this process.

The Dirac concept of a covariant polarized vacuum [74,75] has been marginalized by the physics community because it is believed to conflict with Gauge theory, which is not true because gauge theories are approximations with plenty of room for additional physics. Worse, the Dirac vacuum is troublesome because it is imbibed with extended electromagnetic theory [76-79] bringing in a longitudinal $B^{(3)}$ em-field component with a can of worms filled with photon mass, $m_{\gamma}$ allowing socalled tired light hypotheses. However, it is unlikely there would be Casimir, Zeeman, Sagnac or Aharonov-Bohm effects without it! And as developed here, a Synchronization Backbone with XDLSXD cyclic periodicity demands it.

According to Dirac, the vacuum consists of infinite virtual electrons completely filling the negative portion of the spectrum of a free Dirac operator $D^{0}$. In the presence of a resonant external field, the virtual particles react and the vacuum becomes polarized by;

$$
D^{0}=-i \sum_{k=1}^{3} \alpha_{k} \partial_{k}+\beta:=-i \alpha \cdot \nabla+\beta
$$


where $\alpha=\left(\alpha_{1}, \alpha_{2}, \alpha_{3}\right)$ and $\beta=\left(\begin{array}{cc}I_{2} & 0 \\ 0 & -I_{2}\end{array}\right), \quad \alpha_{k}=\left(\begin{array}{cc}0 & \sigma_{k} \\ \sigma_{k} & 0\end{array}\right)$, with $\sigma_{2}=\left(\begin{array}{cc}0 & -i \\ i & 0\end{array}\right)$, and $\sigma_{3}=\left(\begin{array}{cc}1 & 0 \\ 0 & -1\end{array}\right)[75]$

Developing Feynman's proposal of a synchronization backbone $[59,60]$ is likely the most important construct in this paper. Deriving the proper perspective from the stent given by Feynman on how a UQC might operate - by the imperative of the synchronization backbone. Researchers failed in attempts to create such; seemingly because the modeling was bi-local and at best with a semiclassical line element. QCs must operate simultaneously on the quantum superposition of inputs. While definitive, in practice this has not worked beyond a few qubits because I decoherence time. If reality itself is considered to be a form of $\mathrm{QC}$ at the level of a Dirac covariant polarized vacuum, we discover that a Feynman synchronization backbone is an inherent property in the processing of the XD - LSXD semi-quantum UFM duality. Feynman illustrated his synchronization backbone technique with a 1D 2-body cellular automata with the simple Hamiltonian, $\hat{H} 1 \mathrm{DXY}$. For a periodic system the Hamiltonian took the form, $\hat{H} \equiv \hat{H}_{\text {SYNCH }}=\hat{H} 1 \mathrm{DXY}[59,60]$.

Table 1 attempts to list all pertinent phenomena operating in concert with a Feynman synchronization backbone. It is a speculative model; the hierarchy may not be ordered properly and some elements may be the same as another stated differently. The dramatic point is that the temporal 3-space of the observer is a subspace of a nonlocal atemporal (holographic-like instantaneity) LSXD manifold. The concatenation of phenomena is required for temporal locality-unitarity to surf as it were on the face of nonlocal ubiquity.

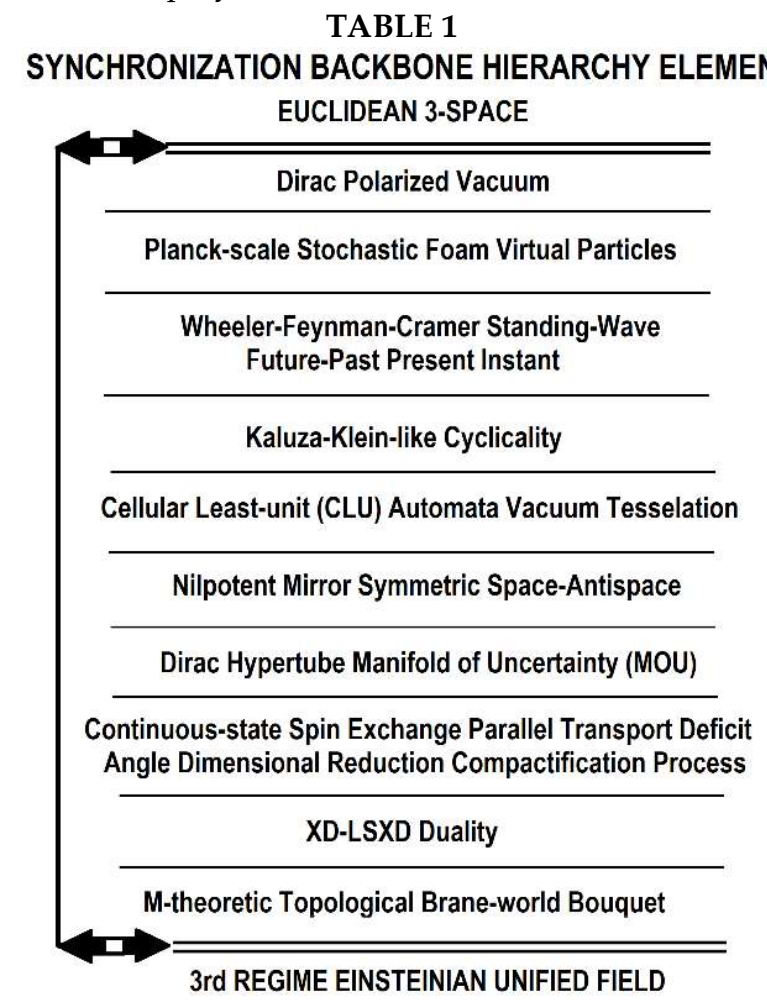

In this continuous state dimensional reduction compactification hierarchy wherein the space of the observer is a sub-manifold, reality cycles from a dual mirror symmetric Calabi-Yau 3-Tori (6D) annihilated by subtractive interferometry from view of the observer. This KK cyclic hierarchy has additional doublings to $12 \mathrm{D}$. This is required because topological elements of matter in this $12 \mathrm{D}$ regime are causally free of the 3-space quantum particle-in-a-box; meaning the image of the mirror image of the mirror image is a causally free ontological panoply of the UF. 


\section{Experimental Protocols - Accessing and Manipulating XD-LSXD}

Novel protocols have been found for empirically testing for XD-LSXD; which if successful could have far reaching consequences for validating M-Theory and provide tabletop-low energy Unified Field Mechanical (UFM) cross section alternatives for viewing putative SUSY partners (or alternative mirror symmetric brane topologies) in a trans-dimensional slice rather than the $\mathrm{TeV}, \mathrm{PeV}$ supercollider techniques utilized/proposed producing standard collision particle sprays as in the highly successful 100 year history of high energy collider physics.

Full TBS XD access requires violation of the quantum uncertainty principle generating a new UFM Transform beyond the Galilean-Lorentz-Poincairé revealing whether one or two additional doublings of a Rowland's type space anti-space model are required for four or five additional TBS spectral lines to complete the radius of the MOU Dirac hypertube. YM-KK equivalence has suggested an empirical path extending SM particle physics. Einstein realized (1905) that Maxwell's equations obey a special relativity principle - Physical law is the same for all observers in uniform relative motion, $x^{\mu}=\left(x^{0}, x^{1}, x^{2}, x^{3}\right)=(t, x, y, z)$. Then, in General Relativity he introduced two indices: $g_{\mu v}(x)$ with line element $d s^{2}=g_{\mu v}(x) d x^{\mu} d x^{v}$. In 1919, Kaluza made his attempt to combine electromagnetism and general relativity by postulating a $5^{\text {th }}$ dimension with an additional HD coordinate, $x^{M}=\left(x^{0}, x^{1}, x^{2}, x^{3}, x^{4}\right)=(t, x, y, z, \theta)$, with line element: $d \hat{s}^{2}=\hat{g}_{M N}(x) d x^{M} d x^{N}$ where he then made a 4D $+1 \mathrm{D}$ split. Klein assumed the 5th dimension had circular topology so that the coordinate, $\theta$ is periodic: $0 \leq \theta \leq 2 \pi$.

The KK $5^{\text {th }}$ dimension was assumed to be curled up at the Planck scale because it was unobserved - As mentioned above this is not the only interpretation for hidden XD.

Now we briefly summarize nine of fourteen proposed protocols; the Main experimental protocol and eight derivatives to test the XD-LSXD continuous-state Long-Range Coherence hypotheses:

1. Basic Experiment - Fundamental test that the concatenation of new OPTFT UFM principles is theoretically sound $[6,24]$. A laser oscillated rf-pulsed vacuum Sagnac resonance hierarchy is set up to interfere with the periodic (continuous-state) structure of an inherent beat frequency within a covariant Dirac polarized spacetime vacuum exciplex to detect the new coherence principle associated with a cyclical holophote entry of the $U F$ into 4 -space. This experiment incursively pokes $a$ hole in spacetime in order to bring the energy of the UF into a detector, essentially discovering a $4^{\text {th }}$ spatial dimension beyond the $3 \mathrm{D}$ of the $\mathrm{SM}$. The remaining protocols are variations of the parameters of this experiment (and basis of all new technologies).

Referring to Fig. 10, imagine a 3-blade ceiling fan symbolic of coordinates forming a quaternion fermion vertex. If one puts one of these fans in front of a mirror (real space) rotating clockwise the mirror image (anti-space) rotates counterclockwise with the blades coming periodically into phase forming the face of a square (Fig. 10). Now we envision how the TBS experiment will work. If a light is put near the fan in real space, i.e. the rf-pulse of our TBS experiment, periodically when the blades come into phase, meaning when a blade from real space comes into conjunction with a blade in the mirror antispace forming a square, the light is reflected off each blade (mirror image of the mirror image) and a pulsating, reflected flash of light occurs in the direction back towards the source/detector representative of how new TBS spectral lines may be discovered - a flash back, like a rotating lighthouse beacon when the resonance hierarchy is aligned properly.

2. Testing for and Manipulating Tight Bound States (TBS) - (similar to protocol 1) Vigier $[66,73]$ has proposed TBS below the 1st Bohr orbit in the Hydrogen atom. Utilizing tenets of the original hadronic form of string theory [3] such as a variable string tension, Ts where the Planck constant, $\hbar$ is replaced with a version of the original Stoney $\lambda[3,84]$, and $\hbar$ is a cyclic asymptote never reached, instead oscillating from virtual Planck to the $\sim$ Larmor radius of the hydrogen atom, i.e. the so-called Planck scale is an apparent compactified restriction imposed by limitations of the Copenhagen Interpretation and is not a fundamental physical barrier. LSXD exist putatively behind the MOU 
barrier of uncertainty and the oscillation of the Planck constant is part of the exciplex gating mechanism [3,6]. Utilizing ontological-phase topological field theory (OPTFT) at the moment of spinspin coupling or spin-orbit coupling an rf-pulse is kicked at various nodes harmonically set to coincide with putative phases in the cycle between localized XD and LSXD cavity TBS properties $[3,12,31,32]$.

3. Bulk Quantum Computing - Utilizing protocol (2) Bulk Scalable UQC can be achieved by superseding the quantum uncertainty principle. (see $[66,73,74,85]$ for details) Programming and data $\mathrm{I} / \mathrm{O}$ are performed without decoherence by utilizing the inherent mirror symmetry properties that act like a Feynman synchronization backbone [3] (Sect. 5) whereby LSXD copies of the local 3-space quantum state are causally free (measurable ontologically by topological switching) without decoherence at specific resonance nodes in the continuous-state conformal Calabi-Yau symmetry cycle hierarchy. A new class of M-theoretic topological qubits and algorithms is required [6].

4. Protein Conformation - (similar to discussion in [86]). Utilizing more macroscopic aspects of protocols $(1 \& 2)$ dual Hadamard quantum logic gates are set as a Cavity-QED spacetime cellular automata $[6,7]$ experiment to facilitate conformational propagation in the prion protein from normal cellular form, PrPc to pathological, PrPSc form by UFM field mediation with the UF force of coherence.

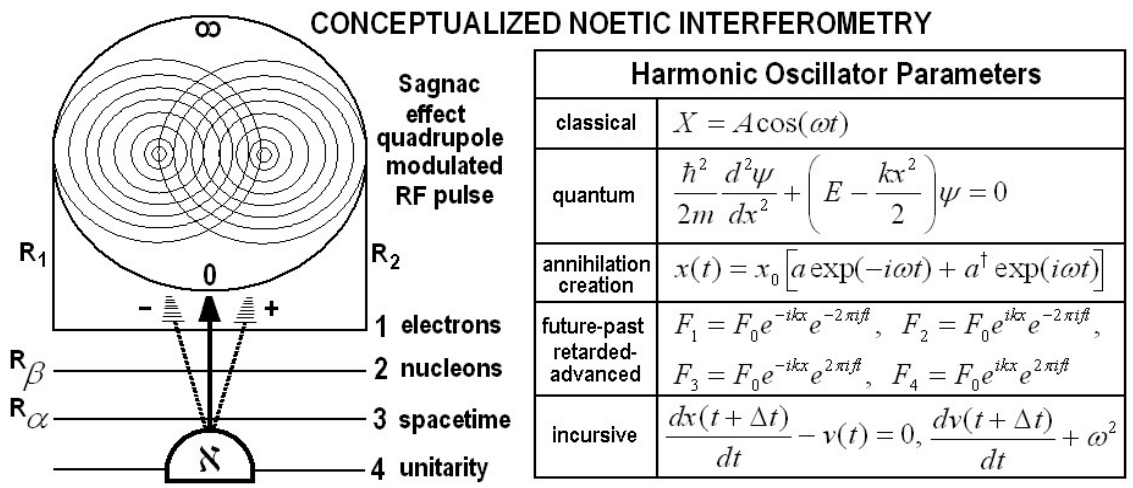

Figure 21. a) Conceptualized cavity-QED multi-level Sagnac effect interferometer designed to punch a hole in spacetime by incursive rf-modulated oscillator to emit the UFM noeon wave, $\aleph$. b) Generalized examples of applied harmonic oscillator - classical, quantum, relativistic, transactional and incursive required to achieve coherent control of the cumulative incursive resonance coupling hierarchy in order to produce harmonic nodes of destructive and constructive interference in the spacetime backcloth. A Bessel-like coupled oscillator with spin-spin coupling hierarchically vibrates electrons coupled to nucleons coupled to spacetime cellular least units opening a hole to XD space.

12D is the minimum required to escape from the temporal bounds of uncertainty, a result of the dimensional tower (Figs. 10,11,13) where time and $E_{3} / M_{4}$ is a standing wave subspace. This structure whether Calabi-Yau, dodecahedral, or some M-theory, F-theory combination entails a reciprocal spiral topology. In this context we utilize logarithmic, helicoid or cyclotron resonance spirals to illustrate new angular momentum commutators. The future-past asymmetry has a Doppler relationship (relative only to the perception of the $4 \mathrm{D}$ observer). $E \& E^{\prime}$, therefore represent equal Wheeler-Feynman future/past symmetries. The Doppler effect arises because of inherent $E$ - $E$ ' boosts and compactifications. In this picture the Wheeler-Feynman-Cramer elements may be understood conceptually by pairs of logarithmic spirals of equal obliquity rolling on a common tangent, $e d$ where each coupled point signifies a present spacetime moment; the locus of which is the arrow of time.

A radiant of spiral, $r$ is

$$
r=a e^{b \theta}
$$


with $a$ the value of $r$ if $\theta=0$, e base of the Naperian logarithms and $b=1 / \tan \phi$, with $\phi$ the constant angle between the tangent to the curve and radiant to the point of tangency. If the value of $\theta$ takes uniform increase (quantized values) the radiants, $r$ will be $c t=0,1,2,3 \ldots, n$ in geometric progression relative to the hierarchal topology of the space. These log curves are not closed; to adapt to continuous motion, pairs must be utilized. Joining corresponding sections of the spiral form symmetrical unilobed wheels. While sectors needn't be equal or symmetrical, the 'wheels' must be paired with sectors of equal obliquity in contact for pure rolling motion to occur. Wheels may also be bilobed or trilobed etc. up to ND to illustrate the Superspace. A tier of three symmetrical wheels.

In the simplistic model of doing the TBS experiment we put hydrogen in a sample tube (Fig. 28) and apply a series of resonant pulses in conjunction with the beat-frequency of space-time to open the HD QED-UFM cavity, send the signal in and allow the new TBS spectral line signal to be emitted back to the detector.

We have postulated that the HD continuous-state peroidicity incorporates cycles of partial commutativity and anti-commutativity until reaching 12D where the 3 -space causal connection is broken. This can be shown metaphorically in terms of logarithmic spirals applied to what is called perfect rolling motion (Fig. 22).

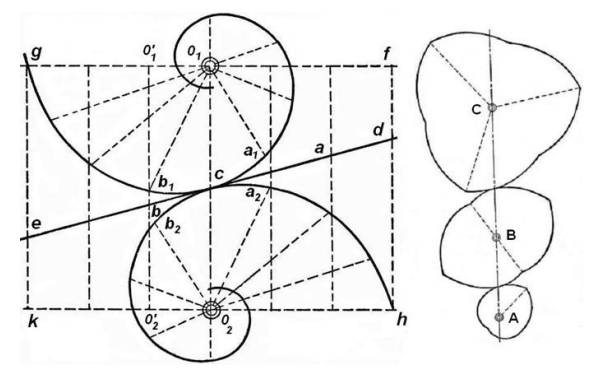

Figure 22. Logarithmic spirals and 'Perfect Rolling Motion. Segments of the logarithmic spiral are put together into the three spheroids on the right, A,B,C. Like the $360^{\circ}-720^{\circ}$ spinor rotation of the Dirac electron; the speroids will only return to the same configuration after a number of $360^{\circ}$ rotations.

The mechanical concept of rolling contact is used to geometrically illustrate the ontological framework for the new LSXDFM commutation rules of angular momentum. A logarithmic spiral coupled to another of the same obliquity undergoes perfect rolling motion (no slippage and constant touching) as long as arcs of the same obliquity coincide. This system of spirals reaches a limit that could be said to be points of Ising flip; but the rotation is not continuous. Making the rolling continuous requires 2 sections of the logarithmic spiral joined into a spheroid. Then continuous motion may occur. This single lobed gear may be made bilobed or trilobed, again for continuous or perfect rolling motion proper obliquity must be maintained.

Here as in the sensory apparatus of the ear (similar principle) the points of contact correspond to frequencies. If the point of contact corresponds to the $z$ axis, we have periodic moments of commutation of angular momentum. Leaving one gear set (the spin tower of frequencies) we have a system of close packed spheres of least cosmological units (CLU) undergoing the UFM mantra (spinexchange, dimensional reduction, compactification) which means that there are HD moments of commutation in the 12D KK-like cyclic structure.

Since angular momentum is the resultant of the atomic magnetic moment and (center of mass) harmonic frequencies (as in the cyclotron frequencies of synchrotron radiation) should make these other ( $\mathrm{x}$ and $\mathrm{y}$ ) components of angular momentum accessible, In any given discretized (composite) $E_{3}$ frame only the $z$ axis will commute as per standard quantum theory; but in the complex HD space the $E_{3}$ non-commutative parameters commute periodically on rotation through mirror tangent nodes of proper obliquity in the continuous state topology; i.e. in considering all HD hyperplanes, there are periodic simultaneous moments where nodes of commutation may be accessible by synchronizing RF pulses of the proper harmonic cyclotron frequency. Bessel functions could be used to manipulate the complex cavity resonance modes. For example, in a generalized Cramer transaction 
event cavity (between future-past topological boundaries) the magnitude of a uniform applied electric field with $E_{0}$ constant can be taken as $E=E_{0} e^{i \omega t}$.

If the frequency increases the electric field flux through any loop $\Gamma_{1}$ produces an oscillating magnetic field $B=i \omega r / 2 c^{2} \cdot E_{0} e^{i \omega t}$ proportional to $r$, the radius of the cavity. This varying magnetic field, proportional to the rate of change of $E$ and thus $\omega$, effects the electric field so it can no longer be uniform by Faraday's Law and also changes with $r$. This requires corrections to our original uniform field $E_{1}$ such that the corrected field must now be $E=E_{1}+E_{2}+E_{3} \ldots E_{n}$ which is best described by the Bessel function $J_{0}$ with

$$
J_{0}(x)=1-\frac{1}{(1 !)^{2}}\left(\frac{x}{2}\right)^{2}+\frac{1}{(2 !)^{2}}\left(\frac{x}{2}\right)^{4}+\frac{1}{(3 !)^{2}}\left(\frac{x}{2}\right)^{6}+\ldots
$$

such that $E$ is now

$$
E=E_{0} e^{i \omega t} J_{0}\left(\frac{\omega r}{c}\right)
$$

Ultimately vacuum technologies will require an advanced M-theoretic UFM form of qubits and algorithms with a new arena for microphysical computation limits - A case of relativistic qubits [5] In the conventional consideration of quantum computing a qubit is any two-state quantum system defined as a superposition of two logical states of a usual bit with complex coefficients that can be mapped to the Riemann sphere by stereographic projection. Formally a qubit is represented as: $\Psi=\xi|0\rangle+\eta|1\rangle$ with each ray $\xi, \eta \in C$ in complex Hilbert space and $\|\Psi\|^{2}=\xi \bar{\xi}+\eta \bar{\eta}=1$, where $|0\rangle$ corresponds to the south or 0 pole of the Riemann sphere and $|1\rangle$ corresponds to the opposite or north or $\infty$ pole of the Riemann complex sphere. The conventional qubit maps to the complex plane of the Riemann sphere as:

$$
\xi \bar{\eta}+\eta \bar{\xi} \rightarrow X, \xi \bar{\eta}-\eta \bar{\xi} \rightarrow i Y, \xi \bar{\xi}-\eta \bar{\eta} \rightarrow Z
$$

Unitary transformations of a qubit correspond to 3D rotations of the Riemann sphere. Following Vlasov for relativistic consideration of a qubit (r-qubit) an additional 4D parameter is added to equation (10):

$$
\xi \bar{\eta}+\eta \bar{\xi} \rightarrow X, \quad \xi \bar{\eta}-\eta \bar{\xi} \rightarrow i Y, \quad \xi \bar{\xi}-\eta \bar{\eta} \rightarrow Z, \quad \xi \bar{\xi}+\eta \bar{\eta} \rightarrow T
$$

In cartography and geometry, the stereographic projection is a mapping that projects each point on a sphere onto a tangent plane along a straight line from the antipode of the point of tangency (with one exception: the center of projection, antipodal to the point of tangency, is not projected to any point in the Euclidean plane; it is thought of as corresponding to a "point at infinity"). One approaches that point at infinity by continuing in any direction at all; in that respect this situation is unlike the real projective plane, which has many points at infinity.

There are essential parameters required of the incursive oscillator to operate properly. The evolution of physical theory from Classical to Quantum changed the fundamental understanding of a point or point particle from continuous - represented in 3D Euclidean space, to discrete fuzzy units with wave-particle duality-represented in 3(4)D Minkowski/ Riemann spacetime. As physical cosmology has evolved towards M-Theory it is now realized that neither of these contexts is sufficient or complete. In UFM Multiverse cosmology the nature of a vertex or point changes into a continuousstate $12 \mathrm{D}$ superspace. This means there are three regimes existing simultaneously/individually: Classical, Quantum and Unity depending on mode of observation. 
Motion of a 1D classical harmonic oscillator is given by $q=A \sin (\omega t+\varphi)$ and $p=m \omega A \cos (\omega t+\varphi)$ where $A$ is the amplitude and $\varphi$ is the phase constant for fixed energy $E=m \omega^{2} A^{2} / 2$. For state $|n\rangle$, with $n=0,1,2, \ldots, \infty$ and with the Hamiltonian $E_{n}=(n+1 / 2) \hbar \omega$ the quantum harmonic oscillator becomes $\left\langle n\left|q^{2}\right| n\right\rangle=\hbar / 2 m \omega\left\langle n\left|\left(a^{\dagger} a+a a^{\dagger}\right)\right| n\right\rangle=E_{n} / m \omega^{2}$ and $\left\langle n\left|p^{2}\right| n\right\rangle=1 / 2(m \hbar \omega)\langle n| a^{\dagger} a+a a^{\dagger}=m E_{n}$ where the terms $a$ and $a^{\dagger}$ are the annihilation and creation operators, $q=\sqrt{\hbar / 2 m \omega}\left(a^{\dagger}+a\right)$ and $p=i \sqrt{m \hbar \omega / 2}\left(a^{\dagger} a\right)$. For the 3D harmonic oscillator each equation is the same with energies $E_{x}=\left(n_{x}+1 / 2\right) \hbar \omega_{x}, E_{y}=\left(n_{y}+1 / 2\right) \hbar \omega_{y}$ and $E_{z}=\left(n_{z}+1 / 2\right) \hbar \omega_{z}$.

In Dubois' notation the classical 1D harmonic oscillator for Newton's second law in coordinates $t$ and $x(t)$ for a mass $m$ in a potential $U(x)=1 / 2\left(k x^{2}\right)$ takes the differential form

$$
\frac{d^{2} x}{d t^{2}}+\omega^{2} x=0 \quad \text { where } \quad \omega=\sqrt{k / m}
$$

which can be separated into the coupled Eqs. (15)

$$
\frac{d x(t)}{d t}-v(t)=0 \quad \text { and } \quad \frac{d v(t)}{d t}+\omega^{2} x=0 .
$$

From incursive discretization, Dubois creates two solutions $x(t+\Delta t) v(t+\Delta t)$ providing a structural bifurcation of the system which together produce Hyperincursion. The effect of increasing the time interval discretizes the trajectory. This represents a background independent discretization of spacetime, a key element in overcoming the usual space quantization.

Each mode of a quantum harmonic oscillator is associated with cavity-QED dynamics, hexagon lattices (Fig. 30) of spacetime topology undergoing continuous transitions. $E$ is the state of energy for $n$ photons. For $n=0$ the oscillator is in the ground state, but a finite energy $1 / 2 \hbar \omega$ of the ground state, called the zero-point energy, is still present in the region of the cavity. According to Eq. (16), the quantum harmonic oscillator field energy of the photons undergoes periodic annihilation and recreation in the periodic spacetime.

$$
E_{n}=\left(n+\frac{1}{2}\right) \hbar \omega .
$$

Nonlocal ontological UFM I/O by supersedes quantum uncertainty. The critical problem in applying conventional QT to the bulk implementation of QC lies in the accompanying theory of measurement; variables observed change destructively in any interaction between particle and observing apparatus. This phenomenological force of interaction is mediated by particle exchange which modifies the Schrödinger equation. In conventional terms 'physical reality is irreducibly quantum' and a qubit resides at a Euclidean, $E^{3}$ or Minkowski, $M^{4}$ vertex.

All attempts for bulk QC have failed in the Copenhagen regime because measurement destroys the quantum system being measured. To overcome this problem the Dirac equation is hyperdimensionalized (complexified) utilizing an extension of Cramer's Transactional Model of QT where all off diagonal elements are physically real and conformally invariant. Bulk implementation of UQC requires a new superspace $N^{12}$ without a real vertex where not only the arbitrary $z$-axis of angular momentum accessible; but the $\mathrm{x}$ and $\mathrm{y}$ components are also real and accessible by a new anticipatory transformation law for ontological evolution utilizing topological switching. This is elucidated by unfolding a hypercube (Figs. 26,27). Relative to the subspace $E_{3}$ the extra square called a satellite is causally free of $E_{3}$ when carried to 12D unitarity. 
During the HD continuous-state topological transformation of the cosmological form of Dirac spherical rotation, a pinch or twist occurs in the middle of the transform followed by an Ising flip of the close-packed complex Riemann spheres which can be driven by the micromagnetic spintronics of fractional and integer quantum Hall effects because of the highly symmetric topological parameters of driven Micromagnetics. This UQC can be implemented in any sufficient multi-state quantum system, whether solid, liquid, bubble, crystal, dot, network, trap, well, vacuum backcloth, comprised of atoms, molecules, ions, photons, spins, NMR, threads, lines, block walls, domain walls, lattices or arrays or topological boundary conditions able to utilize coherent control of the synchronization backbone promoted by Feynman.

In order to avoid the Copenhagen limitations of collapse and dissipation UQC requires utilization of the hierarchical and recursive properties of complex self-organization inherent in the whole universe, not just a portion of its observed parameters. The critical condition is the introduction of a model for evolution of the wave function making correspondence to a new non-collapse (ontological or energyless) version of RQFT. By a coherent control of Ising spin flips of the UFM spacetime least-units (an ontological topological switching of metrics) domains of discretization ( $\Delta x \Delta \rho \cong \hbar)$ may be avoided by utilizing periodic nodes in the resonant hierarchy that are commutative because the Riemann curvature tensor equals zero. E3 is a discretization, a composite of future-past potentials. In HD where the parameters are separated one can manipulate commutative and noncommutative regimes. Another way to illustrate the intended use of coordinated RF-sine wave $\pi$ - pulses with the geometry of spatial rotations of a pair of common dice to show that some rotations commute, $a \otimes b=b \otimes a$ and others are noncommutative $a \otimes b \neq b \otimes a$.

The general equations for putative spacetime exciplex energetics (Fig. 42) are:

$$
\begin{aligned}
& G^{*}+G^{*} \Leftrightarrow Z^{*} ; \quad Z^{*}+m_{\gamma} \Leftrightarrow X^{*} \\
& X^{*}-m_{\gamma} \stackrel{\text { emission }}{\longrightarrow} Z^{*} \text { or } G^{*} \\
& X^{*}+m_{\gamma} \rightarrow Z^{*} \text { or } G^{*}
\end{aligned}
$$

where $G$ is the ZPF ground (not reduced to in excited excimer cycles), Z UFM cavity excited states and $X$ the spacetime C-QED exciplex coupling. The numerous configurations plus the large variety of photon frequencies absorbed could allow for a full C-QED XD-LSXD dual Dirac hypertube absorption-emission equilibrium spectrum. UFM Exciplex Properties hidden behind the veil of uncertainty in antispace entail existence of a harmonic beat frequency as part of the synchronization backbone inherent in the XD-LSXD duality of UFM brane topology. The exciplex complex as illustrated in Fig. 23b and eq. (17) is the programmable mechanism related CLU structure with an inherent KK cyclicality.
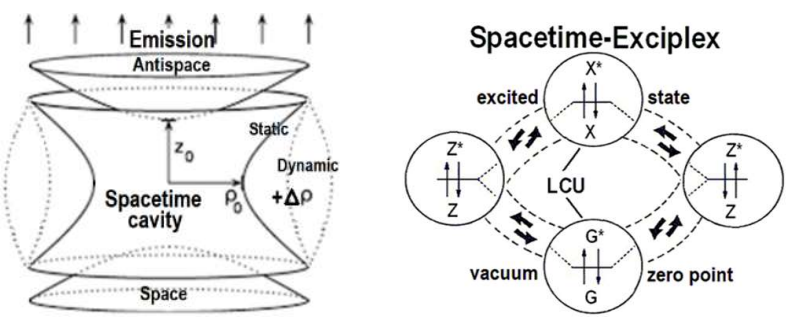

Figure 23. Exciplex UFM spacetime properties. a) Simplistic idealization of a Penning laser trap extended to an antispace static-dynamic oscillating Casimir-Polder cavity incorporating required exciplex energy dynamics. b) Illustrating the energetics of CLU exciplex excitation dynamics in remaining above ground state (eq. 6), putatively allowing UFM energy to continuously summate with a conductive mean-free path. c) Casimir-Polder cavity oscillations also curve space. Fig. 23a) adapted from [87]. 
A torus is generated by rotating a circle about an extended line in its plane where the circles become a continuous ring. According to the equation for a torus, $\left[\left(\sqrt{x^{2}}+y^{2}\right)-R\right]^{2}+z^{2}=r^{2}$, where $r$ is the radius of the rotating circle and $R$ is the distance between the center of the circle and the axis of rotation. The volume of the torus is $2 \pi^{2} R r^{2}$ and the surface area is $4 \pi^{2} R r$, in the above Cartesian formula the $z$ axis is the axis of rotation. Electron charged particle spherical domains fill the toroidal volume of the atomic orbit by their wave motion. If a photon of specific quanta is emitted while an electron is resident in an upper more excited Bohr orbit, the radius of the orbit drops back to the next lower energy level decreasing the volume of the torus in the emission process. We suggest that these toroidal orbital domains have properties similar to QED cavities and apply this structure to topological switching during dimensional reduction in the continuous state multiverse model [2].

When applying the protocol for manipulating UFM cosmology, we extrapolate Einstein's energy dependent or deformed spacetime metric, $\hat{M}_{4}$ to a supersymmetric $12 \mathrm{D}$ standing-wave future-past advanced-retarded multiverse topology for which we have designed a spacetime resonance method for a covariant Dirac polarized vacuum which has properties like an ocean of light. If this is true spacetime acts like a 'surface wave' on the upper regime of the complex self-organized Dirac Sea and is therefore amenable to descriptive methods of nonlinear dispersive wave phenomena generally of the basic form

$$
L(\mu)=\varepsilon N(\mu)
$$

where $L$ and $N$ are Linear and Nonlinear operators respectively in the linear limit where $\varepsilon=0$ with elementary dispersive wave solutions $\mu_{i}=A_{i} \cos \theta_{i}, \theta_{i}=k_{i} x-\omega\left(k_{i}\right) t$ for $1 \mathrm{D}$ plus time where nonlinearity creates resonant interactions between the $\mu_{i}$ solutions and the Amplitude $A_{i}$ depends on $t$, creating potentially substantial effects where initial absent modes can become cumulative interactions producing shock wave effects. Motion of a 1D classical harmonic oscillator is given by $q=A \sin (\omega t+\varphi)$ and $p=m \omega A \cos (\omega t+\varphi)$ where $A$ is the amplitude and $\varphi$ is the phase constant for fixed energy $E=m \omega^{2} A^{2} / 2$. For state $|n\rangle$, with $n=0,1,2 \ldots \infty$ and Hamiltonian $E_{n}=(n+1 / 2) \hbar \omega$ the quantum harmonic oscillator becomes

$$
\left\langle n\left|q^{2}\right| n\right\rangle=\hbar / 2 m \omega\left\langle n\left|\left(a^{\dagger} a+a a^{\dagger}\right)\right| n\right\rangle=E_{n} / m \omega^{2}
$$

and

$$
\left\langle n\left|p^{2}\right| n\right\rangle=1 / 2(m \hbar \omega)\langle n| a^{\dagger} a+a a^{\dagger}=m E_{n}
$$

where $a \& a^{\dagger}$ are the annihilation and creation operators, $q=\sqrt{\hbar / 2 m \omega}\left(a^{\dagger}+a\right)$ and $p=i \sqrt{m \hbar \omega / 2}\left(a^{\dagger} a\right)$.

For the 3D harmonic oscillator each equation is the same with energies

$$
E_{x}=\left(n_{x}+1 / 2\right) \hbar \omega_{x}, E_{y}=\left(n_{y}+1 / 2\right) \hbar \omega_{y} \text { and } E_{z}=\left(n_{z}+1 / 2\right) \hbar \omega_{z}
$$

In Dubois' notation the classical 1D harmonic oscillator for Newton's second law in coordinates $t$ and $x(t)$ for a mass $m$ in a potential $U(x)=1 / 2\left(k x^{2}\right)$ takes the differential form

$$
\frac{d^{2} x}{d t^{2}}+\omega^{2} x=0 \quad \text { where } \quad \omega=\sqrt{k / m}
$$


which can be separated into the coupled equations

$$
\frac{d x(t)}{d t}-v(t)=0 \quad \text { and } \quad \frac{d v(t)}{d t}+\omega^{2} x=0
$$

From incursive discretization, Dubois creates two solutions $x(t+\Delta t) v(t+\Delta t)$ providing a structural bifurcation of the system which together produce Hyperincursion. The effect of increasing the time interval discretizes the trajectory as in Fig. 24 below. This represents a background independent discretization of spacetime.

In a homogeneous magnetic field, the forces exerted on opposite ends of the dipole cancel each other out and the trajectory of the particle is unaffected. if the particles are classical "spinning" particles then the distribution of their spin angular momentum vectors is taken to be truly random and each particle would be deflected up or down by a different amount producing an even distribution on the screen of a detector. Instead, quantum mechanically, the particles passing through the device are deflected either up or down by a specific amount. this means that spin angular momentum is quantized (also called space quantization), i.e. it can only take on discrete values. there is not a continuous distribution of possible angular momenta. this is the usual fundamental basis of the standard quantum theory and where we must introduce a new experimental protocol to surmount it. This is the crux of our new methodology: If application of a homogeneous magnetic field produces quantum uncertainty upon measurement, then "do something else".

In NMR spectroscopy often it is easier to make a first order calculation for a resonant state and then vary the frequency until resonance is achieved. Among the variety of possible approaches that might work best for a specific quantum system, if we choose NMR for the UFM Interferometer it is relatively straight forward to determine the spin-spin resonant couplings between the modulated electrons and the nucleons; but achieving a critical resonant coupling with the wave properties of matter and the spacetime backcloth is another matter. Firstly, for UFM cosmology $\hbar$ is not a rigid barrier as in Standard Model Big Bang-Copenhagen cosmology; $\hbar$ is a virtual limit of past-advanced elements of the continuous-state standing-wave present as it cyclically recedes into the past where the least unit cavities tiling the spacetime backcloth can have radii $\leq$ the Larmor radius of the hydrogen atom.

This new Planck length oscillates through a limit cycle from the Larmor radius of the hydrogen atom to standard $\hbar$. This is like a wave-particle duality - Larmor radius at the future-retarded moment and $\hbar$ at the past-advanced moment. The dynamics are different for future-retarded elements which have been theorized to have the possibility of infinite radius for $\mathrm{D}>4$. This scenario is a postulate of string theory. Considering the domain walls of the least-unit structure, the $\hbar$-Larmor regime is considered internal-nonlocal and the Larmor-infinity regime considered externalsupralocal. For simplicity we introduce our review of NMR concepts for the hydrogen atom, a single proton with magnetic moment $\mu$, angular momentum $J$ related by the vector $\mu=\gamma J$ where $\gamma$ is the gyromagnetic ratio and $J=\hbar I$ where $I$ is the nuclear spin. The magnetic energy $U=-\mu \cdot B$ of the nucleus in an external magnetic field in the $z$ direction is $U=-\mu_{z} B_{0}=-\gamma \hbar I_{z} B_{0}$ where values of $I_{z}, m_{I}$ are quantized according to $m_{I}=I, I-1, I-2, I-3, \ldots-1$.

For most nuclear species the $z$-component of the magnetization, $M$ grows exponentially until reaching equilibrium according to $M_{z}(t)=M_{0}\left(1-e \exp -t / T_{1}\right)$ where $T_{1}$ is the spin-lattice relaxation time. Of interest for the UFM interferometer is the fact that as $\mu$ precesses cyclically from $m_{I}=-1 / 2$ to $m_{I}=+1 / 2$ the nucleons experience a torque, $\tau$ changing $J$ by $\tau=d J / d t$ or $\mu \times B=d J / d t$. Under thermal equilibrium the x-y components are zero; but $M_{z}$ can be rotated into the x-y plane creating transverse $M_{x}$ and $M_{y}$ components $d M / d t=\gamma M \times B$ for the entire system by applying a rotating circularly polarized oscillating magnetic field $2 B_{1} \cos \omega t \hat{i}$ of frequency $\omega$ in addition to the constant magnetic field $B_{0} \hat{k}$. Now the total time dependent field decomposes 
into the two counterpropagating fields

$$
B_{1}(\cos \omega t \hat{i}+\sin \omega \hat{t})+. B_{1}(\cos \omega t \hat{i}-\sin \omega t \hat{j})
$$

This more complicated form for use with multiple applied fields is necessary, as described below, for use with the Sagnac Effect, quadrupole, and dipole dynamics required to operate the UFM interferometer. Nuclear Quadrupole Resonance (NQR) is a form of NMR in which quantized energy level transitions are induced by an oscillating RF-magnetic field in the electric quadrupole moment of nuclear spin systems rather than the magnetic dipole moment. The nuclear quadrupole moment, $Q$ is based on the nuclear charge distributions $\rho(r)$ departure from spherical symmetry defined as the average value of $1 / 2\left(3 z^{2}-r^{2}\right) \rho(r)$ over the nuclear volume. $Q$ has the dimension of area where the nuclear angular momentum, for which $m_{I}=I$ where $I$ is the nuclear spin quantum number and $m_{I}$ is the quantum number for the $z$ component of the spin $m_{I}=-1,+1, \ldots, I-1, I$.

Nuclei with $I=0$ have no magnetic moment and are therefore magnetically inert. Similarly, in order for $Q=0$ the nucleus must be spherical with spin $I \geq 0$. For spin $I=1 / 2$ nuclei have dipole moments, $\mu$ but no $Q . Q$ is positive for prolate nuclei and negative for oblate nuclei. For an isolated nucleus in a constant magnetic field $H_{0}$ with nuclear spin number $I>0$ the nucleus possesses a magnetic moment. From Quantum Theory (QT) the length of the nuclear angular momentum vector is $[I(+I)]^{1 / 2} \hbar$ where measurable components are given by $m \hbar$ with $m$ the magnetic quantum number taking any $(2 I+I)$ value from the series $I, I-I, I-2, \ldots,-(I-I),-I$. For the $I=3 / 2$ case there are four values along the direction of the applied magnetic field $H_{0}$. Of the three types of spinspin coupling, this experiment relies the hyperfine interaction for electron-nucleus coupling, specifically the interaction of the nuclear electric quadrupole moment induced by an applied oscillating RF-electric field to act on the nuclear magnetic dipole moment $\mu$. When the electron and nuclear spins are strongly aligned along their $z$-components the Hamiltonian is $-m \cdot B$, and if $B$ is in the $z$ direction

$$
H=-\gamma_{N} I \cdot B=-\gamma_{N} B I_{x}
$$

with $m=\gamma_{N} I, \gamma_{N}$ the magnetogyric ratio $\gamma_{N}=e \hbar / 2 m_{p}$ and $m_{p}$ the mass of the proton.

Radio frequency excitation of the nuclear magnetic moment, $\mu$ to resonance occurs for a nucleus collectively which rotates $\mu$ to some angle with respect to the applied field $B_{0}$. This produces a torque $\mu_{i} \times B_{0}$ causing the angular momentum, $\mu$ itself to precess around $B_{0}$ at the Larmor frequency $\omega_{L}=\gamma_{N} B_{0}$. This coherent precessing of $\mu$ can also induce a 'voltage' in surrounding media, an energy component of the Hamiltonian to be utilized to create interference in the structure of spacetime. Metaphorically this is like dropping stones in a pool of water: One stone creates concentric ripples; two stones create domains of constructive and destructive interference. Such an event is not considered possible in the standard models of particle physics, quantum theory and cosmology. However, UFM science uses extended versions of these theories wherein a new teleological action principle is utilized to develop what we call a 'transistor of spacetime'. Just as standard transistors and copper wires provide the basis for almost all modern electronic devices; This Laser Oscillated Vacuum Energy Resonator (LOVER) using the information content of spacetime geodesics (null lines) will become the basis of many forms of UFM Technologies.

Simplistically in this context, utilizing an array of modulated tunable lasers, atomic electrons are RFpulsed with a resonant frequency that couples them to the magnetic moment of the nucleons such that a cumulative interaction is created to dramatically enhance the Haisch-Rueda inertial backreaction [88-92]. The laser beams are counter-propagating producing a Sagnac effect Interferometry to maximize the violation of Special Relativity. This is the $1^{\text {st }}$ stage of a multi-tier experimental platform designed (according to tenets of UFM Theory) to open a hole in the fabric of spacetime in 
order to isolate and utilize the force $\hat{F}_{U}$ of the Unified Field.

The interferometer utilized as the basis for our vacuum engineering research platform is a multitiered device. The top tier is comprised of counter-propagating Sagnac effect ring lasers that can be built into an IC array of 1,000+ ring lasers. If each microlaser in the array is designed to be counterpropagating, an interference phenomenon called the Sagnac Effect occurs that violates special relativity in the small scale. This array of RF modulated Sagnac-Effect ring lasers provides the top tier of the multi-tier LOVER Inside the ring of each laser is a cavity where quantum effects called Cavity Quantum Electrodynamics (C-QED) may occur. A specific molecule is placed inside each cavity, a xanthene quantum dot, or prion protein in our sample embodiments. If the ring laser array is modulated with resonant frequency modes chosen to achieve spin-spin coupling with the molecule's electrons and neutrons, by a process of Coherent Control of Cumulative Interaction an inertial backreaction is produced whereby the electrons also resonate with the spacetime backcloth in order to 'open an oscillating hole' in it. This requires a form of RQFT compatible with the 12D version of Mtheory called F-Theory relying on symmetry conditions of UFM cosmology within which it is cast.

The first step in the interference hierarchy (Fig. 25) is to establish an inertial back-reaction between modulated electrons and their coupled resonance modes with nucleons. The complete nature of inertia remains a mystery. But if one follows the Sakarov and Puthoff conjecture, the force of gravity and inertia, the initial resistance to motion, are actions of the vacuum zero-point field. Therefore, the parameter $m$ in Newton's 2nd law $f=m a$ is a function of the zero-point field. Newton's $3^{\text {rd }}$ law states that 'every force has an equal and opposite reaction'. Haisch \& Rueda claim vacuum resistance arises from this reaction force, $f=-f$. We derived an electromagnetic interpretation of gravity and electro-magnetism that suggests this inertial back-reaction is like an electromotive force of the de Broglie matter-wave field in the spin exchange annihilation creation process inherent in a hysteresis of relativistic spacetime fabric. In fact, we go further to suggest that the energy responsible for Newton's $3^{\text {rd }}$ law is a result of the continuous-state flux of the ubiquitous UFM field. For the LOVER we assume the Haisch-Rueda postulate is correct

$$
f=\frac{d \rho}{d t}-\lim _{\Delta t \rightarrow 0} \frac{\Delta \rho}{\Delta t} \equiv \frac{d \rho_{*}}{d t_{*}}-\lim _{\Delta t_{*} \rightarrow 0} \frac{\Delta \rho_{*}}{\Delta t_{*}}=f_{*}
$$

where $\Delta \rho$ is the impulse given by the accelerating agent and thus $\Delta \rho_{*}^{z p}=-\Delta \rho_{*}$.

The cyclotron resonance hierarchy must also utilize the proper beat frequency of the continuousstate dimensional reduction spin-exchange compactification process inherent in the symmetry of UFM spacetime naturally tuned to make the speed of light $c \equiv c$. With this apparatus in place UFM theory suggests that destructive-constructive C-QED interference of the spacetime fabric occurs such that the UFM eternity wave, $\aleph$ of the unified field, $U_{F}$ is harmonically (like a holophote) released into the cavity of the detector array. Parameters of the Dubois incursive oscillator are also required for aligning the interferometer hierarchy with the beat frequency of spacetime. As illustrated in Fig. 25 the coherent control of the multi-level tier of cumulative interactions relies on full utilization of the continuous-state cycling inherent in parameters of UFM cosmology. What putatively will allow UFM interferometry to operate is the harmonic coupling to periodic modes of Dirac spherical rotation in the symmetry of the HD geometry. The universe is no more classical than quantum as currently believed; reality rather is a continuous state cycling of nodes of classical to quantum to unified, $C \rightarrow Q \rightarrow U$. The hierarchy of UFM cosmology is cast in 12D such that the pertinent form of relativistic quantum field theory (RQFT) has significantly more degrees of freedom whereby the modes of resonant coupling may act on the structural-phenomenology of Dirac sea itself rather than just the superficial zero-point field surface approaches to vacuum engineering common until now.

The parameters of the UFM oscillator may best be implemented by RQFT using a form of de Broglie fusion. According to de Broglie a spin 1 photon can be considered a fusion of a pair of spin $1 / 2$ corpuscles linked by an electrostatic force. Initially de Broglie thought this might be an electronpositron pair and later a neutrino and antineutrino: "A more complete theory of quanta of light must 
introduce polarization in such a way that to each atom of light should be linked an internal state of right and left polarization represented by an axial vector with the same direction as the propagation velocity". These prospects suggest a deeper relationship in the structure of spacetime of the Cramer type. The epistemological implications of a 12D RQFT must be delineated. The empirical domain of the SM relates to the $4 \mathrm{D}$ phenomenology of elementary particles. It is the intricate notion of what constitutes a particle that concerns us here - the objects emerging from the quantized fields defined on Minkowski spacetime. This domain for evaluating physical events is insufficient for our purposes. The problem is not only the additional degrees of freedom and the associated extra-dimensionality, or the fact that 'particles' can be annihilated and created but that in UFM cosmology they are continuously annihilated and recreated within the holograph as part of the annihilation and recreation of the fabric of spacetime itself. This property is inherent in the 12D Multiverse because temporality is a subspace of eternity. This is compatible with the concept of a particle as a quantized field. What we are suggesting parallels the wave-particle duality in the propagation of an electromagnetic wave. We postulate this as a property of all matter and spacetime albeit as continuous-state standing waves. For a basic description, following de Broglie's fusion concept, assume two sets of coordinates $x_{1}, y_{1}, z_{1}$ and $x_{2}, y_{2}, z_{2}$ which become

$$
X=\frac{x_{1}+x_{2}}{2}, Y=\frac{y_{1}+y_{2}}{2}, Z=\frac{z_{1}+z_{2}}{2}
$$

Then for identical particles of mass $m$ without distinguishing coordinates, the Schrödinger equation (for the center of mass) is

$$
-i \hbar \frac{\partial \psi}{\partial t}=\frac{1}{2 M} \Delta \psi, \quad M=2 m
$$

Equation (28) corresponds to the present and Eq. (29a) corresponds to the advanced wave and (29b) to the retarded wave.

$$
-i \hbar \frac{\partial \phi}{\partial t}=\frac{1}{2 M} \Delta \phi, \quad-i \hbar \frac{\partial \varphi}{\partial t}=\frac{1}{2 M} \Delta \varphi
$$

Extending Rauscher's concept for a complex 8- space differential line element $d S^{2}=\eta_{\mu v} d Z^{\mu} d Z^{* v}$, where the indices run 1 to $4, \eta_{\mu \nu}$ is the complex 8-space metric, $Z^{\mu}$ the complex 8-space variable and where $Z^{\mu}=X_{\mathrm{Re}}^{\mu}+i X_{\mathrm{Im}}^{\mu}$ and $Z^{* v}$ is the complex conjugate, to $12 \mathrm{D}$ continuous-state UFM spacetime; we write just the dimensions for simplicity and space constraints

$$
x_{\mathrm{Re}}, y_{\mathrm{Re}}, z_{\mathrm{Re}}, t_{\mathrm{Re}}, \pm x_{\mathrm{Im}}, \pm y_{\mathrm{Im}}, \pm z_{\mathrm{Im}}, \pm t_{\mathrm{Im}}
$$

where \pm signifies Wheeler-Feynman/Cramer type future-past/retarded-advanced dimensions.

This dimensionality provides an elementary framework for applying the hierarchical harmonic oscillator parameters.
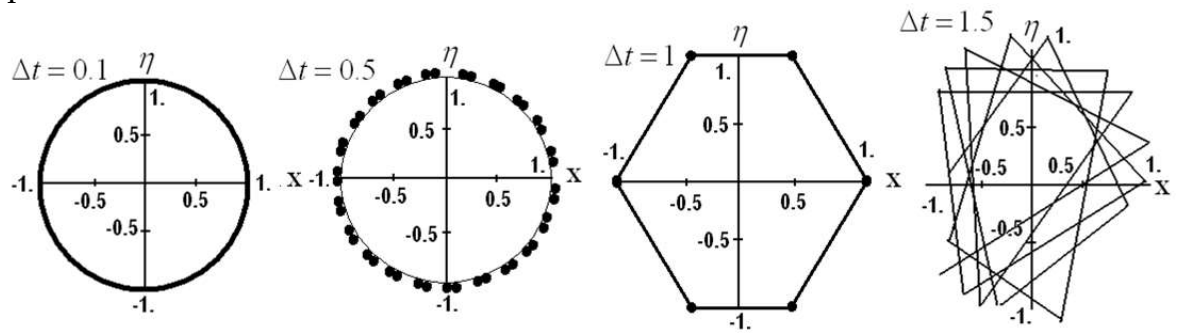

Figure 24. Numerical phase space trajectory simulations for Dubois' superposed incursive oscillator $x_{n}=1 / 2\left[x_{n}(1)+x_{n}(2)\right], v_{n}=1 / 2\left[v_{n}(1)+v_{n}(2)\right]$ based on coordinates and velocities shown for values 
of $\Delta \tau=\omega t$ equal to $0.1,0.5,1.0$ and 1.5. Initial conditions are $\chi_{0}=1, \eta_{0}=0$ and $\tau_{0}=0$ with total simulation time $\tau=\omega t=8 \pi$. Key element of resonance hierarchy.

We have all the pieces to formulate a new UF transform group, but are not yet sufficiently aware of topological restrictions to make a formal attempt; it is not clear in this case, whether experiment or theory will drive discovery. Because of the importance of this looming condition, we take liberty to speculate, hypothetically outlining the plethora of required components. As noted above our version of UFM M-theory reverts to an original hadronic form of string-theory having a tachyon (considered nonphysical) and variable string tension. Both these concepts become relevant in the UFM scenario where the present instant is a standing-wave of the future-past. Indeed the 1945, Wheeler-Feynman absorber theory,

$$
T_{\text {tot }}(\mathrm{X}, t)=\sum_{n}\left(E_{n}^{\mathrm{ret}}(\mathrm{X}, t)+E_{n}^{\mathrm{adv}}(\mathrm{X}, t)\right) / 2+\sum_{n}\left(E_{n}^{\mathrm{ret}}(\mathrm{X}, t)-E_{n}^{\mathrm{adv}}(\mathrm{X}, t)\right) / 2=\sum_{n} E_{n}^{\mathrm{ret}}(\mathrm{X}, t)
$$

describes radiation as a standing wave [21].

Cramer's transactional interpretation (TI) of quantum mechanics (based on Wheeler-Feynman absorber theory), also describes quantum interactions as standing waves formed by retarded (forward-in-time) and advanced (backward-in-time) waves [21]. Many consider Cramer's TI standing-waves too primitive; but a 1D oscillating string is only the basic concept. In reality, when extended to Calabi-Yau mirror symmetric dual 3-tori, a 6D hyperspherical standing wave M-theoretic topological interaction; the model can be made to work.
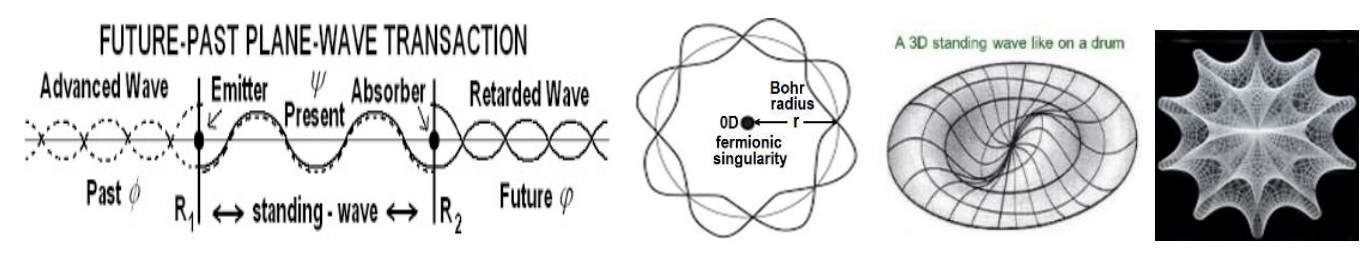

Figure 25. Cramer's Transactional Interpretation showing 1D, 2D, 3D and 6D standing waves.

For Space-antispace, the nilpotent condition for the two vector spaces is made from arbitrary scalar values and represented by the 5 generator objects $E, p_{x}, p_{y}, p_{z}, m$ to form the two commuting vector spaces,

$$
\left(\mathrm{K} E+i \operatorname{Iil} p_{x}+i \operatorname{Ij} p_{y}+i \mathrm{Ik} p_{z}+i \mathrm{Jk} m\right)\left(\mathrm{K} E+i \operatorname{Ii} p_{x}+i \operatorname{Ij} p_{y}+i \mathrm{Ik} p_{z}+i \mathrm{Jk} m\right)=0
$$

Which becomes the nilpotent condition, $E^{2}-p_{x}^{2}-p_{y}^{2}-p_{z}^{2}-m^{2}=0$. The bracketed object above has squared to zero, the duality is identical and defines the principle of a point in either space as a norm 0 crossover between them. Physics is mediated by the concept of space, requiring a dual space to ensure that the fundamental condition of the universe is a zero totality. The real space of observation is defined by quaternions as i, j, k [34]. The dual space, I,J,K not accessed as a physical quantity (until now) is called 'vacuum space', or 'antispace' because it combines with real space to produce a nilpotent zero totality. The creation of nilpotent structures zeroing all higher terms and the perfect group symmetry allowing a complete cancellation ensure that Nature exhibits zero totality in all of its aspects, material and conceptual, and it does this via a fundamental principle of duality.

Another way to look at this is to say the fermion always exists in the two spaces from which it is constructed, real space and vacuum space, and the non-classical zitterbewegung motion, Schrödinger found in the solution to the free-particle Dirac equation, represents the switching between these spaces which makes it possible to define the fermion as creating a point singularity through the intersection of two spaces. We can here apply a reverse argument from topology. The creation of a 
particle singularity using its 'intersection' with a dual space can be seen as the creation of a multiplyconnected space from a simply-connected space through the insertion of a topological singularity.

According to a well-known argument, parallel transporting a vector round a complete circuit in a multiply-connected space will produce a phase shift of $\pi$ or $180^{\circ}$ in the vector direction, whereas transporting it round a simply-connected space will not, and so, in the first case, the vector will be required to do a double circuit to return to its starting point [6]. This is exactly what happens with a spin $1 / 2$ fermion, which, as a point-singularity, can be regarded as existing in its own multiplyconnected space. We can interpret this as meaning that the fermion requires a double circuit because, just as in zitterbewegung, it spends only half of its time travelling in the real space of observation.

The first step in the interference hierarchy (Fig. 26) is to establish an inertial back-reaction between the modulated electrons and their coupled resonance modes with the nucleons. The complete nature of inertia remains a mystery [93]. But if one follows the Sakarov [94] and Puthoff [95] conjecture, the force of gravity and inertia, the initial resistance to motion, are actions of the vacuum zero-point field. Therefore, the parameter $m$ in Newton's second law $f=m a$ is a function of the zeropoint field [96-98]. Newton's third law states that 'every force has an equal and opposite reaction'. Haisch \& Rueda [88-92] claim vacuum resistance arises from this reaction force, $f=-f$. We have also derived an electromagnetic interpretation of gravity and electromagnetism [99] that suggests this inertial back-reaction is like an electromotive force, force, $E$ where the internal resistance $r$ generated when a load is put upon an electric current $I$ between a potential difference $V$, i.e. $r=(E-V) / I$. The de Broglie matter-wave field in the spin exchange annihilation creation process inherent in a hysteresis of relativistic spacetime fabric (Fig. 9). In fact, we go further to suggest that the energy responsible for Newton's third law is a result of the continuous-state flux of the ubiquitous UFM field. For the L.O.V.E.R. we assume the Haisch-Rueda postulate is correct

$$
f=\frac{d \rho}{d t}-\lim _{\Delta t \rightarrow 0} \frac{\Delta \rho}{\Delta t} \equiv \frac{d \rho_{*}}{d t_{*}}-\lim _{\Delta t_{*} \rightarrow 0} \frac{\Delta \rho_{*}}{\Delta t_{*}}=f_{*}
$$

where $\Delta \rho$ is the impulse given by the accelerating agent and thus $\Delta \rho_{*}^{z p}=-\Delta \rho_{*}[88-92]$.

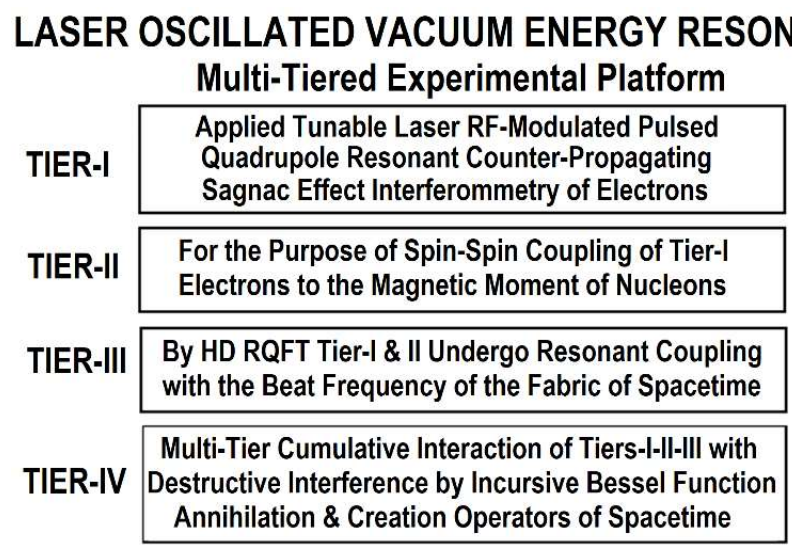

Figure 26. Design elements for the HD Cavity-QED trap of the UFM Interferometer postulated to constructivelydestructively interfere with the topology of the 12D spacetime manifold for manipulation of the unitary field. Substantial putative effects are possible if a cumulative interaction of the interference nodes of the cyclotron resonance hierarchy is set up to produce shock waves.

The cyclotron resonance hierarchy must also utilize the proper beat frequency of the continuousstate dimensional reduction spin-exchange compactification process inherent in the symmetry of UFM spacetime naturally 'tuned' to make the speed of light $c \equiv c$. With this apparatus in place UFM theory suggests that destructive-constructive C-QED interference of the spacetime fabric occurs such 
that the noeon eternity wave, $\aleph$ of the unitary field, $U_{F}$ is harmonically (like a holophote) released into the cavity of the detector array. Parameters of the Dubois incursive oscillator are also required for aligning the interferometer hierarchy with the beat frequency of spacetime.

In Fig. 26 design element Sagnac effect RF pulsed resonance hierarchy are applied to the quantum conditions for resonant coupling to the HD Cavity-Exciplex trap of the Interferometer to constructively-destructively coherently control the topology of the 12D spacetime manifold to manipulate the unitary field. Cumulative effects are made by coupling constructive spacetime interference nodes of the cyclotron resonance hierarchy.
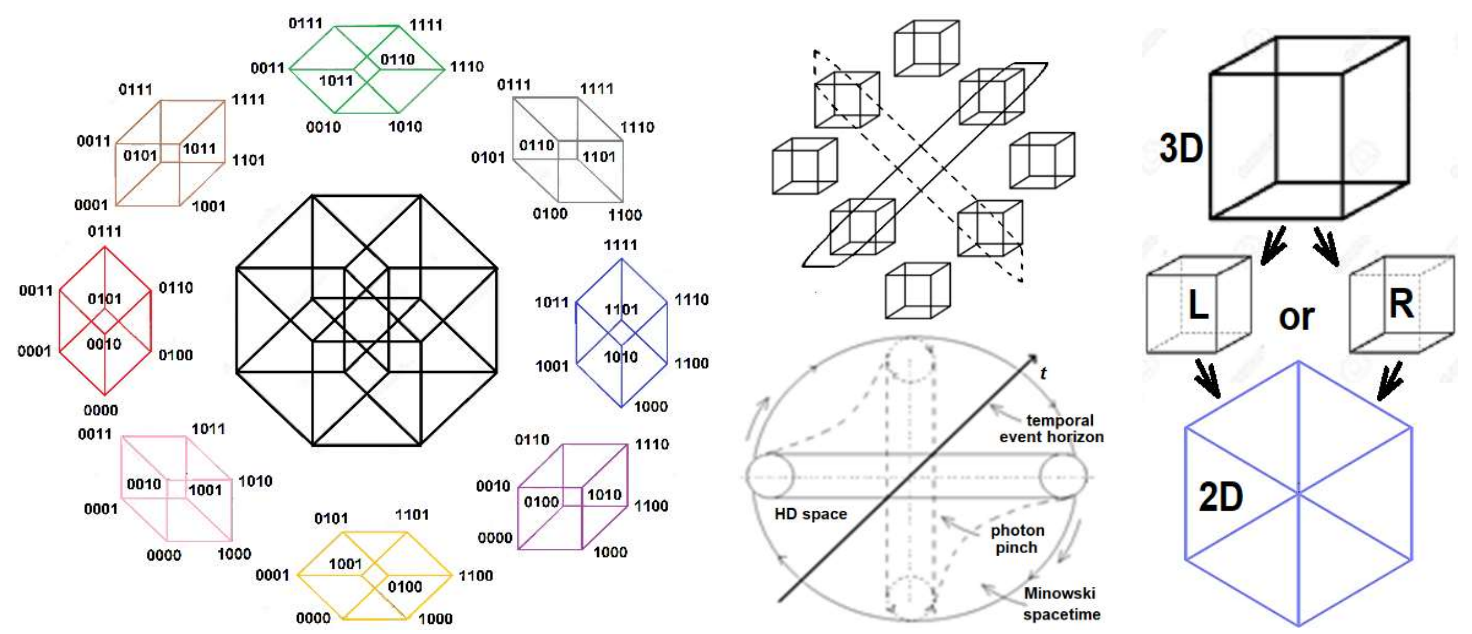

Figure 27. a) Exploded 4D hypercube, 64 overlapping vertices (4) or 16 subsets $(16 \times 4=64)$. b-top) Exciplex configuration of CLU array. One of four orthogonal configurations, with mirror symmetry another four. In terms of developing the required noetic transform, each 3-cube represents an ambiguous Necker cube able to perform topological raising and lowering moves. The four cubes illustrated have interplaying vertex points, dotted 0101:1011 and 0100:1010; solid - 0110:1101 and 0010:1001. b-bottom) Conceptualized view of the HD quadrupole photon-vacuum complex for quadrupole $\Leftrightarrow$ dipole interactions as elements of the UF at the event horizon of Minkowski spacetime. c) Dimensional reduction from L-R ambiguous Necker cube.

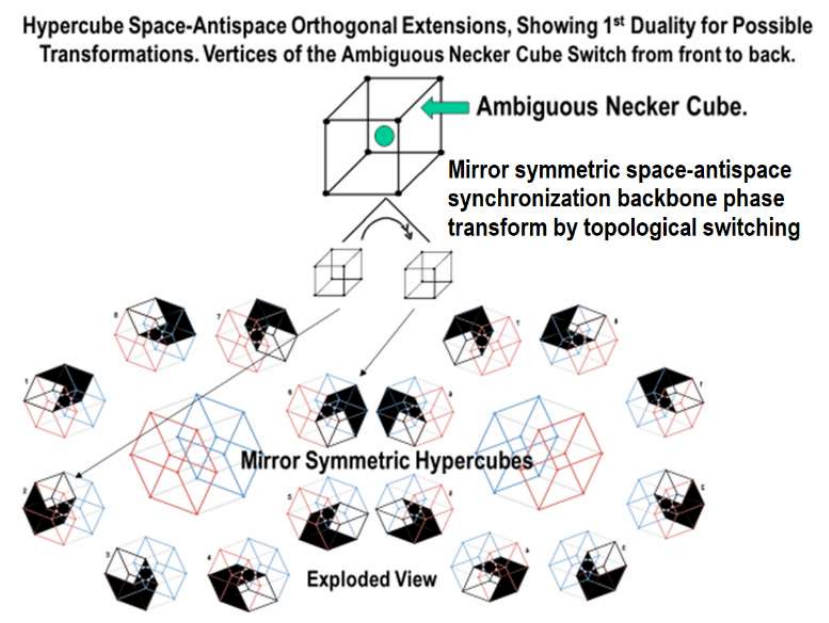

Figure 28. Example of how the periodicity of cyclic supersymmetry or mirror symmetric CLU rotation elements act in dimensional phase transformations by topological switching in boosts and compactification.

5. Manipulating special case of the Lorentz Transformation. Aspects of a spacetime exciplex model $[2,6]$ in terms of restrictions imposed by Cramer's Transactional Interpretation [21] on mirror 
symmetry can be used for the putative detection of virtual tachyon-tardyon interactions in zitterbewegung [33].

Hypersphere Volumes

\begin{tabular}{|l|l|c|}
\hline$D(n)$ & Volume & Volume $r=1$ \\
\hline 0 & 0 & 0 \\
\hline 1 & 2 & 2 \\
\hline 2 & $\pi \mathrm{r}^{2}$ & 3.14159 \\
\hline 3 & $4 / 3 \pi \mathrm{r}^{3}$ & 4.18879 \\
\hline 4 & $1 / 2 \pi^{2} \mathrm{r}^{4}$ & 4.93480 \\
\hline 5 & $8 / 15 \pi^{2} \mathrm{r}^{5}$ & 5.26379 \\
\hline 6 Degenerete? & $\infty$ \\
\hline
\end{tabular}

Table 2: Standard Hypervolume values for increasing $n$-dimensionality of MOU radius, $r$ for unit sphere or $n$ ball equal to 1. If LSXD exist, degeneracy would occur at the MOU hypertube limit of $r$ discovered in the same manner the outermost energy level of an atom is detected when the outermost electron acquires sufficient energy to escape to infinity. Hypersphere volumes which can be used to calculate new TBS spectral lines.

In usual spectroscopy, the first two spectral lines of hydrogen occur at $.5 \AA$ and $2 \AA$ suggesting arbitrarily in terms of Table 2, that three additional TBS hyperspherical spectral lines might occur between that range (calculation not performed) at. $8 \AA, 1.1 \AA$ and $1.4 \AA$ for example, to the MOU hypertube limit, whereafter a putative $4^{\text {th }}$ TBS spectral line would be degenerate, meaning the input signal would enter LSXD space and fly to infinity.
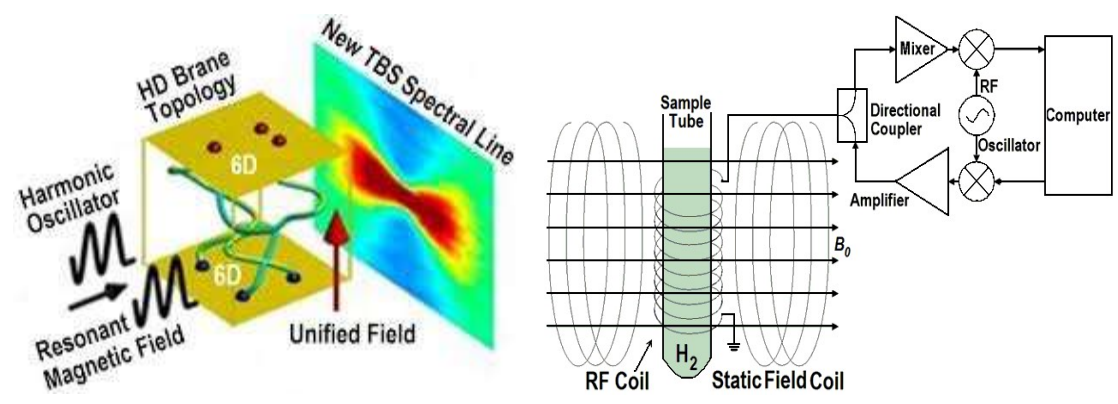

Figure 29. Illustrations of basic TBS protocol. a) Through incursive resonance oscillator signal interferes with the 6D space-antispace MOU cavity. If the signal strikes the hyperspherical XD domain wall, as in usual spectroscopic analysis, the signal returns as a new TBS spectral line. b) Simplified experimental NMR-like resonance apparatus for putative LSXD C-QED ionization of TBS in hydrogen. The Fig. only shows possible details for rf-modulating TBS QED resonance, not the spectrographic recording and analysis components.

6. Extended Quantum Theory - Test of causal properties of de Broglie- Bohm-Vigier quantum theory by utility of the UF holophote effect (protocol 1 parameters) as a super quantum potential to summate by constructive interference the density of de Broglie matter waves.

7. Coherent Control of Quantum Phase - Additional test of the de Broglie- Bohm interpretation for existence of a nonlocal pilot wave - quantum potential for manipulating the phase space quantization in a double slit experiment by controlling which slit quanta passes through. Application to quantum measurement and transistor lithography refinement.

8. Manipulating Spacetime CLU Structure - (similar to protocol 6) Test of conformal scaleinvariant properties of a putative Dirac conformal polarized vacuum, possible continuous-state property related to an arrow of time (Also similar to basic experiment, but more advanced).

9. Test for the UFM Unique String Vacuum - Until now the structure of matter has been explored by building ever bigger supercolliders like the CERN LHC. If the LSXD access model in 
terms of a Dirac covariant polarized energy dependent vacuum proves correct utilizing the inherent conformal scale-invariant mirror symmetry properties of de Broglie matter waves will allow examining various cross sections in the structure of matter in symmetry interactions during cyclic continuous-state future-past annihilation-creation modes of matter in the CLU tessellated spacetime metric without the need for supercolliders. There are a number of very specific postulated cosmological properties required in order to perform these experiments.

We were able to devise a 2D computer simulated 3-brane production of a Dirac hypertube with a rf-pulsed oscillator configuration to facilitate opening and closing its radius. It was assumed that hexagonal configurations of spacetime are significant in manipulating the Dirac vacuum. Autodesk Chaos Software was utilized in running the simulation [107].

From the proof of Schöenflies theorem [108] there can be no topological knots in a plane. Therefore, there can be no topological torsion in a 2D reality. According to tenets of M-Theory matter remains on the 2-brane and gravity is free to pass between branes. A simulated creation of an F-Theory 2brane from the Dirac polarized vacuum [74,75] is demonstrated utilizing the Autodesk Chaos Software [107].

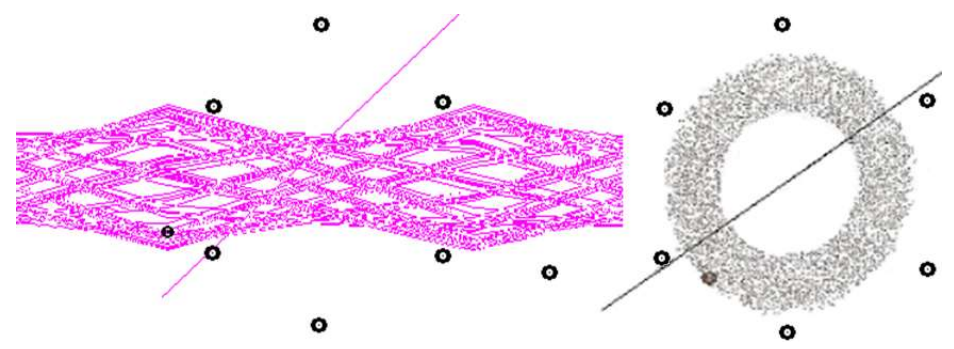

Figure 30. Two views of one form of computer simulated production of a 2-brane from parameters of the hexagonal geometry of the putative Dirac polarized vacuum. Hysteresis loop harmonic oscillation of the future-past dynamics produces branes by incursive resonance. The software simulation of 2-brane emergence from the geometry of spacetime least-units is achieved by applying a harmonic oscillator generated by the energy of the UFM Action Principle. The oblique lines in each figure are insertion angles and the two tiny points are the holophote injection points of noeon energy.

\section{Table 3. SPACETIME HARMONIC OSCILLATOR PARAMETERS}

PARAMETER

Charge

Magnetic Capture Radius

Magnetic Field Radius

Pull Towards Center

Frequency

Friction (String Tension)
VALUE USED

3

5

11

27

33

1.37
POSSIBLE RANGE

$\pm 500$

0 to 20

1 to 60

$\pm 500$

2 to 10,000

0 to 500

Susceptible to being perturbed. Van der Waals forces quickly vanish at longer distances between interacting molecules (or spacetime CLU tessellations). Van der Waals forces are of the same origin as the Casimir effect, arising from quantum interactions with the zero-point field [109]. The resulting van der Waals forces can be attractive or repulsive [110]. An example of parametric oscillation is pumping on a playground swing. A person on a moving swing can increase the amplitude of the swing's oscillations without any external drive force (pushes) being applied, by changing the moment of inertia of the swing by rocking back and forth (pumping) or alternately standing and squatting, in rhythm with the swing's oscillations. The varying of the parameters drives the system.

The Casimir-Polder interaction between a single neutral atom and a nearby surface, arising from 
the (quantum and thermal) fluctuations of the electromagnetic field, is a cornerstone of cavity quantum electrodynamics (C-QED), and theoretically well established. Recently, Bose-Einstein condensates (BECs) of ultracold atoms have been used to test the predictions of C-QED. The purpose of the present thesis is to upgrade single-atom C-QED with the many-body theory needed to describe trapped atomic BECs. Tools and methods are developed in a second-quantized picture that treats atom and photon fields on the same footing. We formulate a diagrammatic expansion using correlation functions for both the electromagnetic field and the atomic system. The formalism is applied to investigate, for BECs trapped near surfaces, dispersion interactions of the van der WaalsCasimir-Polder type, and the Bosonic stimulation in spontaneous decay of excited atomic states.

What this means is that when constructive resonance occurs, information of state is ontologically transferred. Spin-spin or spin-orbit coupling can only transfer information under proper commutativity conditions. When q-uncertainty is in a topological phase state (exclusion principle) only destructive interference may occur. When sufficient mirror symmetric phase is applied, such that the $n$th state is causally free of the local C-QED state, the ontological phase transition may then summate (transfer information) as a resonance of constructive interference.

The at present putative ontological phase transition is a LSXD M-theoretic form on the well-known 5D K-K cyclicality. It is the inherent cyclicality of the M-theoretic brane topology that allows both periodic commutative violation of the uncertainty principle and the ontological transfer of information relative to the quantum state. Interaction free measurement entails a quantum Zeno effect because it cannot fully escape or cheat uncertainty and not bypass the semiclassical limit of the standard model in $4 \mathrm{D}$. But measurement at the semi-quantum limit may under stringent phase periodicity perform an ontological interaction of $P \equiv 1$.

Traditionally electron standing-waves oscillate about the atomic nucleus. Here we attempt to expand the wave nature of matter itself as static waves centered on the locus of least spacetime units as it is annihilated and recreated in the arrow of time relative to the observer. This requires a conversion of the de Broglie wave equation, $m v r=n(h / 2 \pi)$ to a static form amenable to the parameters of continuous-state cosmology [2]. For Hyperspherical Representation the magnitudes of the radial coordinates of a two-state wavefunction, $\psi\left(\vec{r}_{1}, \vec{r}_{2}\right)$ in hyperspherical representation are replaced by the hyper-spherical radius, $R$ and the hyperspherical angle, $\alpha$ such that $R \equiv\left(r_{1}^{2}+r_{2}^{2}\right)^{1 / 2}$ and $\alpha \equiv \arctan r_{2} / r_{1}$ in order that the symmetries may be more clearly shown. The hyperspherical radius, $R$ represents the size of the 2-state system and the hyperspherical angle, $\alpha$ is a measure of the radial correlation of the two-state system [2]. It is critical to note that when $\alpha=\pi / 4, r_{1}=r_{2}$; and when $\alpha=0$ or $\pi / 2$ one of the states is at a greater distance from the leastunit vertex than the other.

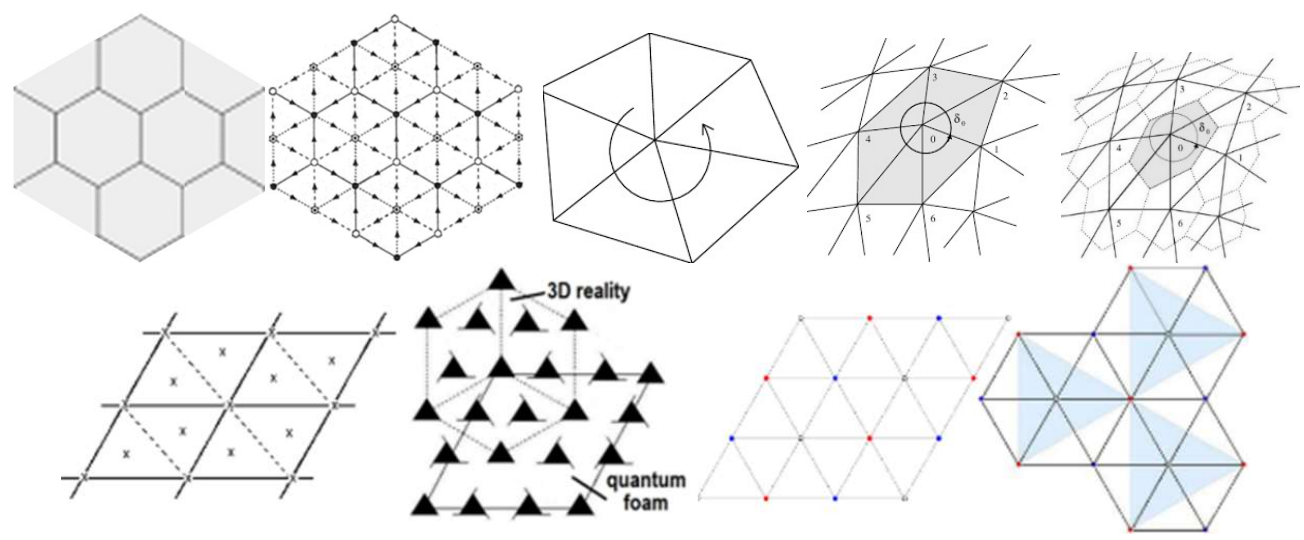

Figure 31. Periodic hexagon tiling. a) 2D plane. b) 3D Spacetime with restricted micromagnetic interactions. c) D eficit angle parallel transport 3D to 2D. d,e) Embedding in spacetime CLUs. f) Hexagonal embedding. g) Boost from 0D Planck foam to 3D. h) Hexagonal trefoil. i) Trefoil boost 2D to 3D. 
In regards to Fig. 31, 2D and projected 3D view of CLU tiling, giving rise to higher HD in the spacetime backcloth. CLU spacetime loses its stochasticity at the semi-quantum limit; and becomes more ordered by coherent control at the upper (HD) limit of the manifold of uncertainty by coherence of the UF. These are CLU exciplex C-QED backcloth tessellations of space, able to accommodate any geometry and any transform by topological switching.

\section{Beyond Electroweak-Strong Interaction Symmetry - Metatron Cube of Platonic Solids}

Electroweak scale Supersymmetry (SUSY) has been popular over its 40-year development history as a likely candidate to ameliorate the hierarchy problem within Gauge theory without any fine-tuned quadratic divergency cancellations in perturbation theory. More recently strong SUSY is being explored in ATLAS experiments. However, our choice aligns with an XD-LSXD duality. This LSXD ADD model (Arkani-Hamed, Dimopoulos, Dvali), attempts to solve the hierarchy problem by postulating our 4D universe exists on a submanifold of 11D M-theoretic space. The natural forces (electromagnetic, strong, weak) operate within the 4D membrane, but gravity operates across all 11D branes, suggesting why gravity is weak compared to the other fundamental forces [111].

The Glashow-Salam-Weinberg Electroweak unification introduced self-similar geometric symmetry structures producing a renormalizable Yang-Mills field spontaneous symmetry breaking mechanism for the $\mathrm{SU}(2) \times \mathrm{U}(1)$ gauge group for Standard Model $\mathrm{W}^{ \pm}$and $\mathrm{Z}^{0}$ bosons [112-115].

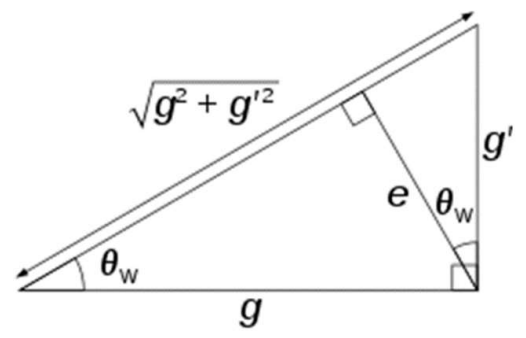

Figure 32. Weinberg weak mixing angle, $\theta_{W}$ with coupling constants, $g, g^{\prime}, e$; adapted from [116].

The spontaneous symmetry breaking mechanism causes the $W_{3}$ and $B$ bosons to coalesce into the $Z^{0}$ boson and a photon, $\gamma$.

$$
\left(\begin{array}{c}
\gamma \\
Z^{0}
\end{array}\right)=\left(\begin{array}{cc}
\cos \theta_{\mathrm{W}} & \sin \theta_{\mathrm{W}} \\
-\sin \theta_{\mathrm{W}} & \cos \theta_{\mathrm{W}}
\end{array}\right)\left(\begin{array}{c}
B \\
W_{3}
\end{array}\right)
$$

with $\theta_{\mathrm{W}}$ the weak mixing angle where the particle axes have been rotated in the $\left(W_{3}, B\right)$ plane by angle $\theta_{\mathrm{W}}$.

Here we take a cursory look at a weak-strong SUSY alliance and LSXD hierarchy problem in terms of terms of a coincidence of all 5 Platonic solids. Because as suggested in protocol 9 above, the concept becomes experimentally testable in terms of a new class of XD-LSXD low energy room temperature table top cross sections that could reveal the geometric topology of string configurations comprising the structure of subatomic particles and atomic nuclei.

A menagerie of electromagnetic strong mixing angles restricted by mass-charge, angular momentum "Electromagnetic strong force" in 2003 Vigier briefly mentions, with no attempt at correspondence, the Platonic solids [117]. I recently discovered the Metatron cube, a coincidence of all 5 Platonics, the idea is that a particle element can exist at each vertex. With the Weinberg electromagnetic mixing angle in mind (elevated to a 6D topological manifold, in a 12D Einstein space) with myriad more mixing angles for the strong force (spacetime tessellation), I think it might be possible to find correlated restrictions to describe a fundamental string for M-theory for the 1st time!

It doesn't appear that quaternions provide a sufficient nilpotency to complete a 12D UFM 
regime. A configuration called the Octonion snowflake model allows the octonions fold into a cube, I believe it will take Qs and Os to close the Einstein UFM space [118].

Regarding the location of protons and neutrons with extended nuclei, the construction of "tight" nuclei with many protons and neutrons, is still an open problem. We only attempt to discuss it within our model, in terms of consequences of extended non-zero electromagnetic charges existing within protons and neutrons in the presence of new short-range spin zero Coulomb type-forces existing within protons and neutrons. Present theories of such nearby forces suggest that protons (and neutrons) repel each other up to very close distance and then a binding force takes hold which forces them to stick together. If this distance is small enough neighbor protons (and neutrons) interact directly.

Assuming:

1) That the electromagnetic charges (in extended protons and neutrons) can first be considered as point-like i.e. representing their interactions by two different types of elements. They interact through the spin (zero part of the) Maxwellian electromagnetic four vector potentials $A_{\mu}$ associated to a nonzero photon mass $m_{\lambda}$ i.e., with static forces in the $\mathrm{keV}$ region corresponding to the spin- 0 component of the Coulomb field.

2) That the equilibrium situation between protons (and neutrons) in given extended nuclei is calculated (first approximation) at point-like charges and leaves aside the fact that charges oscillate at velocities $\vec{v} \simeq c$, around associated centers of mass: i.e., a calculation which neglects their space extension and is, performed in the rest-frame of the centers of mass. This amounts to consider protons (and neutrons) as charged points and to work in the special Lorentz frame where we consider the protons and neutrons in our model as containing three em Charges \pm 1 or two em charges $(+1$ and -1 + neutrinos) so that the neutron mass is slightly superior in the reaction $n \rightarrow \rho+e^{-}+\bar{r}$.

3) That in Moon's model [119] the neutron and proton subelements are not located at random. They are located at the angles of the five known 3D Platonic solids built with equal length between their point angles which lie at equal distance on the surface of a 3D sphere (circumsphere) and having only one sphere inscribed (insphere) within them. They are defined by Table 3 and Fig. 33.

TABLE 4 - Platonic Solids

\begin{tabular}{|l|l|l|l|}
\hline Polyhedron name & Vertices & Faces & Edges \\
\hline Tetrahedron & 4 & 4 & 6 \\
\hline Cube & 8 & 6 & 12 \\
\hline Octahedron & 6 & 8 & 12 \\
\hline Icosahedron & 12 & 20 & 30 \\
\hline Dodecahedron & 20 & 12 & 30 \\
\hline METATRON CUBE & $\mathbf{5 0}$ & $\mathbf{5 0}$ & $\mathbf{9 0}$ \\
\hline
\end{tabular}

Table 4. Geometric elements of the five Platonic solids and their coincidences in the Metatron cube.

Table 4 shows the 50 nested vertices (Metatron cube) with space-antispace mirror symmetric standing-wave duality seems sufficient to putatively begin to contemplate how to conceptualize the string/brane basis of particle physics and inherent strong interaction UFM mixing angles. Eventual utility of the TBS protocol provides a new type of low energy table-top (sans supercollider) cross sections methodology for testing M-theoretic elementary particle dynamics. The uncertainty principle comes into play twice here: it determines the range of the strong nuclear force, and it 
determines the size of the smallest possible space.

4) These five Platonic solids define a type of limit of what can be perfectly constructed in 3D space. These solids are the only ones that can be formed with faces that are equal, regular plane figures (the equilateral triangle, square and pentagon) and equal solid angles. A derivative set of solids, the semiregular or Archimedean solids, can thus be formed using two or three regular plane figures for faces in each figure. Both species of solids can be circumscribed by a sphere, the circumsphere, such that all the vertices of the figure are just touched by the sphere. The platonic solids are unique in that each has just one sphere, the isosphere, that will sit inside, just tangent to the interior of each one of the Metatron's 50 faces.

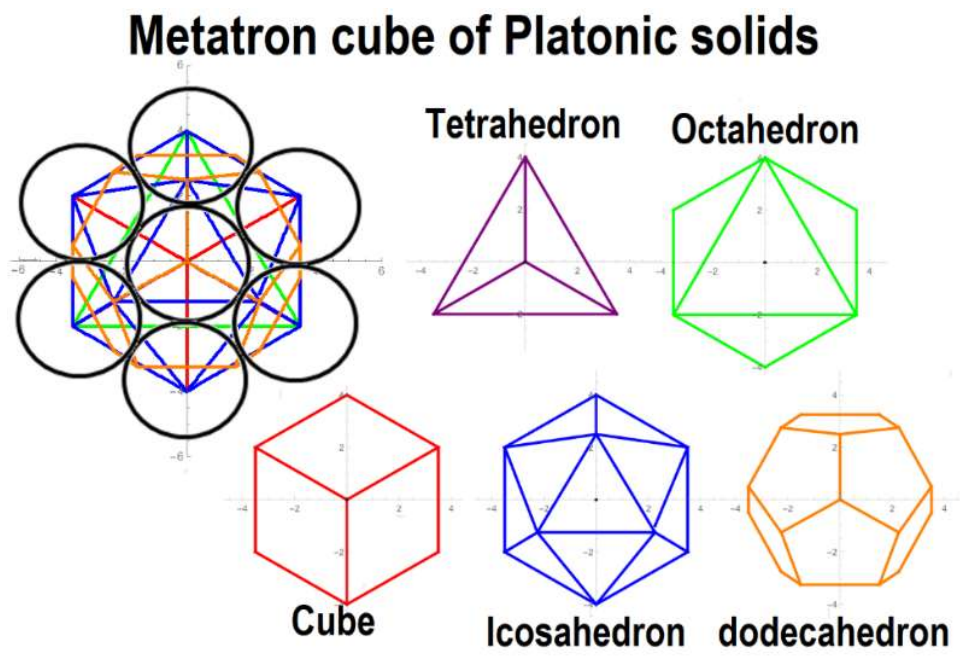

Figure 33. The Metatron cube, a superimposed assembly of the five Platonic solids on a close packed Cellular Least Unit assembly tessellating spacetime. Adapted from Wolfram [120].

The Archimedean solids must have either two or three distinct inspheres. A third series of spheres, the midspheres is formed by a radius connecting the center of the solid with the midpoint of each of the edges: It is associated with both the Platonic and Archimedean solids.

The surfaces of the platonic solids and related regular solids represent unique divisions of the surface of a sphere according to a least action principle.

5) That in this type of "Keplerian Atom" the 92 elements of the naturally occurring elements are determined by two identical sets of nested solids, each containing 46 vertices. This justifies the Mendeleev Table.

Two pairs of regular platonic solids (cube-octahedron and isocahedron-dodecahedron) arranged in a nested sequence. The tetrahedron being dual unto itself.

These 3-dimensional geometrical figures have been utilized to explain the observed periodicity of the atomic volumes (the ratio of atomic weight/density) of the elements. The maxima of atomic numbers, $3,11,19,37,55$ and 87 identify the elements that begin each period of the Mendeleev Table where minima occur at, or near, the atomic numbers $8,14,26,46$ which mark the completed proton shells.

Protons and neutrons have a slightly different mass and their electromagnetic charges (around each angle) move at velocities close to $c$ in internal protons and neutrons. They can only lie at extremities of the 3D volumes with centers separated by specific distances: In Moon's model the tight charges are thus separated by different distances. They lie at different angles (points) of the vertices of two identical pairs of nested solids in 3D.

Assuming the location of the 92 observed protons and neutrons of the naturally observed elements are located at vertices of two identical pairs of nested solids: they are thus comparable to Platonic and 
Archimedean solids. This description is, of course, only an approximation, in our model, since the proton and neutron carry three and two moving electromagnetic charges at $\vec{v} \simeq c$ and thus have a slightly different mass and internal geometry. If one works in their center of mass rest frame where their volume is essentially spherical, their electromagnetic point-like charges are located in a neutron at opposite sides of a neutron's diameter.

6) Since observed periodic properties of the elements (compressibility, coefficient of expansion, and reciprocal melting points) obey the same periodicity as atomic volume in this model, the abrupt change in nuclear properties happen around Magic Numbers in nuclear properties. This also explains why the observed periodicity of physical and chemical properties of nuclei is not the same as the periodicity of the proton shells inside the nuclei [119].

A path to new physics may be provided for by the Strong Interaction applied to the vertices of the Platonic solids as mixing angles for string / brane topological phase transitions. The field in which a particle is embedded carries the force exerted by the particle mediating its motion through space, the range of which is limited by the Uncertainty Principle, $\Delta E \Delta t \geq h / 4 \pi$, limited to the Planck time, $\Delta t \approx \geq h / 4 \pi \Delta E$. This process cannot be detected within the current status of field theory. $\Delta E$ is associated with a mass, $\Delta E=m c^{2}$, limiting the distance the mediating field particle can travel to $d \approx c \Delta t$.

This Metatron cube scenario is highly speculative and axiomatic at this point in development; but to us seems logically sound and internally consistent. Further, if our group is not the one to perform the experiments; it seems of high importance to allow the concepts an arena for debate in the hopes that progress may be accelerated.

\section{Discussion}

From the 60 Years of Yang-Mills Gauge Field Theories conference, I fondly recall Nobel Laureate Davis Gross' comment to me during my presentation on Tight Bound States (TBS): “Some crazy experiment..." [121]. I supremely appreciating the necessity of rigor, but alas it is too possible to misinterpret precision results by myopic expectations. For example, recalling the biopic film of Steven Hawkins life, he said, "who is God to tell me how to write my equation." Summarizing Hossenfelder: We have not seen progress since the 1970s in foundations of physics since the SM of particle physics was formed. Ever since, theories describing observations have remained unchanged. The last confirmed particle, the Higgs-boson, was predicted in the 1960s. But shortcomings - no quantization of gravity, dark matter, quantum measurement problem, etc. known for more than 80 years are as unsolved today as they then [122].

No paradigm shift has occurred for over a hundred years, or new physics for 40 or 80 years depending on how one calculates. We suspect three salient reasons: 1) Myopic insistence on a quantum gravity - not the regime of integration. 2) If additional dimensions exist, they must be curled up at the Planck scale because they are not observed - not the only interpretation. 3) Ignoring the existence of a Dirac polarized vacuum because it is believed it interferes with Gauge Theory - not so, Casimir, Zeeman, Sagnac and Aharonov-Bohm effects, for all practical purposes, demonstrate the existence of a Dirac covariant polarized vacuum. This scenario justifies a Radical Divergence from Current Thinking; therefore, license has been taken to present radical views, attempting to help induce the imminent paradigm shift.

Feynman claimed gravity may not be quantized [123]; recent Nobel Laureate Roger Penrose spoke more clearly: people think you should quantize GR and this would give you some kind of crazy foam structure... My view for the combination of QM with GR you need a marriage. That marriage will not be a QG in the general sense of the word a QG. It will have to be a theory where QM itself is modified because QM is a self-inconsistent theory. QT can't survive at all levels; it consists of 2 parts. 1) evolution of the quantum system, the Schrödinger equations unitary evolution where the state evolves in time which is an indeterminate equation. 2) what happens when you take a measurement, 
a completely different theory. The measuring device is supposed to be treated classically according to standard Copenhagen point of view, which doesn't make sense. Why does the quantum system suddenly choose one of the alternatives available? So, there is something missing in the theory itself, the thing missing has to do with gravity. There are conflicts between QM and GR, resolving those conflicts will result in a change in QM [124]. In contrast to Penrose we go one step further; QM is not complete so of course it will be falsified at the semi-quantum limit, however, we propose that there is no QG (except a virtual form at the semi-quantum limit) because the arena of integration is with a $3^{\text {rd }}$ regime of natural science - that of an Einsteinian UFM.

I apologize to reviewer(s) of this paper for the license incautiously taken for radical postulates; but the author believes they are logical and delineate sufficient correspondence to current theory, just not in a popular manner. It goes without saying, the panoply proposed is empirically testable; that is what the paper is promulgating; and in spite of problems the issues are in dire need of debate.

Conflicts of Interest: The author declares no conflict of interest.

Funding: Please add: "This research received no external funding"

\section{References}

1. Huerta, J. Schreiber, U. M-theory from the Superpoint, 2018; arXiv:1702.0177

2. Amoroso, R.L. Rauscher, The Holographic Anthropic Multiverse: Formalizing the Complex Geometry of Reality. 2009; 43. World Scientific: London.

3. Amoroso, R.L. Paradigm for a continuous-state holographic conscious multiverse. R.L. Amoroso, B. Lehnert J-P Vigier Eds. Beyond the Standard Model: Searching for Unity in Physics. 2005; 332-351 The Noetic Press: Oakland; https://vixra.org/pdf/2010.0085v1.pdf.

4. Dirac, P.A.M. Classical theory of radiating electrons, Proc. Roy. Soc. London A 1938; 167, 148-169.

5. Amoroso, R. L. Vigier, J. P. The Dirac electron hypertube revisited nonlocal parameters within extended particle elements. Fundamental Physics at The Vigier Centenary: "L'Hérétique de la Physique" Lives On, 2020; World Scientific, London, (in press).

6. Amoroso, R.L. Universal Quantum Computing: Supervening Decoherence-Surmounting Uncertainty, 2017; World Scientific: London.

7. Osoroma, D.S. A programmable cellular automata polarized Dirac vacuum, 71-80. RL Amoroso et al. Eds. The Physics of Reality: Space, Time, Matter, Cosmos, 2013; 504-509. World Scientific: Singapore.

8. Toffoli, T. Margolus, N.H. Invertible cellular automata: a review. Physica D: Nonlinear Phenomena, 1990; 45(1-3), 229-253.

9. Zizzi, P. Spacetime at the Planck scale: The quantum computer view, R.L. Amoroso et al.(Eds. Fundamental Physics at The Vigier Centenary: "L'Hérétique de la Physique" Lives On, 2020; 430-450 World Scientific, London, (in press).

10. Dragić, A. Marić, Z. Vigier, J-P New quantum mechanical tight bound states and cold fusion experiments. Physics Letters A, 2000; 265(3), 163-167.

11. Dragić, A. Marić, Z. Vigier, J-P. On the possible existence of tight bound states in quantum mechanics. in Gravitation and Cosmology: From the Hubble Radius to the Planck Scale 2002; 349-356). Springer: Dordrecht.

12. Amoroso R L Vigier J-P Evidencing tight bound states in the hydrogen atom Empirical manipulation of large-scale XD in violation of QED The Physics of Reality Space Time Matter Cosmos-Proc 8th Symp Honoring Math Physicist Jean-Pierre Vigier, R L Amoroso et al. Eds. 2013; World Sci: London; http://vixra.org/pdf/ 1305.0053v2.pdf.

13. Vigier, J-P New hydrogen (deuterium) Bohr orbits, 1993; Proceedings ICCF4, Hawaii, 4, 7.

14. Bhabha, H.J. Corben, H.C. General classical theory of spinning particles in a Maxwell field, Proceed Royal Society of London Series A: Mathematical, Physical \& Engineering Sciences 1941; 178:974, 273-314.

15. Barut, A.O. Surv. High Energy Phys 1980; 1, 113.

16. Chantler, C.T. et al. Testing three-body electrodynamics with trapped $\mathrm{Ti}^{20+}$ ions: evidence for a z-dependent divergence between experiment and calculation Phys Rev Let 2012; 109153001.

17. Chantler, C.T. Discrepancies in quantum electro-dynamics. Radiation Phys and Chem 2004; 71,611-617.

18. Chantler, C.T. Laming, J.M. Silver, J.D. Dietrich, D.D. Mokler, P.H. Finch, E.C. Rosner, S.D. Phys. Rev. A, 2009; 80, 022508.

19. Anagnostopoulos, D.F. Gotta, D. Indelicato, P. Simons, L.M. Phys. Rev. Lett. 2003; 91, 240801.

20. von Neumann, J. The Mathematical Foundations of Quantum Mechanics, 1955; Princeton Univ. Press: 
Princeton.

21. Cramer, J.G. The transactional interpretation of quantum mechanics, Rev. Mod. Phys. 1985; 58, 647-687; https://www.researchgate.net/profile/John_Cramer2/publication/280926546_The_transactional_ interpretation_of_quantum_mechanics/links/59aaf2fea6fdcce55a34bfba/The-transactional-interpretationof-quantum-mechanics.pdf; Wheeler, J.A. Feynman; R.P. Interaction with the absorber as the mechanism of radiation Rev Mod Phys, 1945; 17, 2-3 157-181.

22. Rowlands, P. How many dimensions are there? R. L. Amoroso et al. Eds. Unified Field Mechanics: Natural Science Beyond the Veil of Spacetime, 2016; 46-54, World Scientific: Singapore; https://www.researchgate.net/ profile/Peter_Rowlands/publication/301432637_How_Many_Dimensions_are_There/links/ 582ddee208ae102f072db0c2.pdf.

23. Amoroso, R.L. Rauscher, E.A. Derivation of the string tension formalism from inherent parameters of a holographic anthropic multiverse, Cs Varga, I. Dienes, R.L. Amoroso Eds. Unified Theories, 2008; 35-46, The Noetic Press: Oakland; https://vixra.org/pdf/1305.0107v1.pdf.

24. Amoroso, R.L. Einstein/Newton duality: An ontological-phase topological field theory, Journal Phys: Conf Series, 2018; 1051,1, 012003. IOP Publishing: London.

25. Amoroso, R.L. Newton-Einstein G-Duality and Dirac-Majorana fusion modeling as mediated by ontological-phase topological field theory, Unified Field Mechanics II, 2017; Singapore: World Scientific: Singapore.

26. Stevens, H.H. Size of a least unit, M. Kafatos Ed. Bell's Theorem, Quantum Theory and Conceptions of the Universe, 1989; Kluwer Academic: Dordrecht.

27. Barvinsky, A.O. Quantum geometrodynamics: The Wheeler-DeWitt equations for the wave function of the universe. Phys. Lett., B, 1986; 175(4), 401-404.

28. Feng, J.C. Matzner, R.A. From path integrals to the Wheeler-DeWitt equation: Time evolution in spacetimes with a spatial boundary. 2017; https://arxiv.org/pdf/1708.07001.pdf.

29. Reifler, F. Morris, R. Conditions for exact equivalence of Kaluza-Klein and Yang-Mills theories, 2007; (arXiv:0707.3790 [gr-qc].

30. Amoroso, R.L Yang-Mills Kaluza-Klein equivalence: An empirical path for extending the standard model. R.L. Amoroso, L.H. Kauffman, P. Rowlands Eds. Unified Field Mechanics: Natural Science Beyond the Veil of Spacetime, 2015; World Scientific: London.

31. Amoroso, R.L. "Shut the front door!": Obviating the challenge of large-scale extra dimensions and psychophysical bridging, R.L. Amoroso, L.H. Kauffman, P. Rowlands. Eds. The Physics of Reality: Space, Time, Matter, Cosmos, 2013; World Scientific: Singapore.

32. Amoroso, R.L. Simple resonance hierarchy for surmounting quantum uncertainty, R.L. Amoroso, P. Rowlands, S. Jeffers. Eds. AIP Conference Proceedings, 2010; 1316, 1,185-193.

33. Amoroso, R.L. Rauscher, E.A. Empirical protocol for measuring virtual tachyon/tardon interactions in a Dirac vacuum, R.L Amoroso, P. Rowlands, S. Jeffers Eds. Search for Fundamental Theory, 2010; AIP Conf. Proc. 1316, 1, 199.

34. Rowlands, P. The Foundations of Physical Law, 2014; World Scientific, Singapore.

35. Bailin, D. Love, A. Kaluza-Klein theory, 1987; Rep. Prog. Phys. 50, 1087-1170.

36. Becker, K. Becker, M. Schwartz, J.H. String Theory and M-Theory: A Modern Introduction, 2007; Cambridge Univ. Press: Cambridge.

37. O'Raifeartaigh, L. The Dawning of Gauge Theory, 1997; Princeton Univ. Press: Princeton.

38. Witten, E. Solutions of four-dimensional field theories via M-theory, Nuc Phys B, 1997; 500, 1-3, 3-42.

39. Banks, T. A critique of pure string theory: Heterodox opinions of diverse dimensions, 2003; arXiv: hepth/0306074.

40. Martin, S.P. A supersymmetry primer, 2011; arXiv:hep-ph/9709356v6.

41. Gogberashvili, M. Four dimensionality in non-compact Kaluza-Klein model, 1999; ArXiv:hep$\mathrm{ph} / 9904383 \mathrm{v} 1$.

42. Overduin, J.M. Wesson, P.S. Kaluza-Klein gravity. Physics Reports, 1997; 283, 303-378.

43. Witten, E. Search for a realistic Kaluza-Klein theory, 1981; Nuclear Physics B 186, 412-428.

44. Kaluza, T. Zum unitätsproblem in der physik, 1921; Sitzungsber, Preuss. Akad. Wiss. Berlin. Math. Phys. 966972.

45. Klein, O. Quantentheorie und funfdimensionale relativitatstheorie, 1926; Zeits. Phys. 37, 895.

46. Klein, O The atomicity of electricity as a quantum theory law, Nature, 1926; 2971, 118, 516.

47. Sundrum, R. SSI lecture notes part 2, 2005; (http://www.slac.stanford.edu/econf/C0507252/lecnotes/ Sundrum2/sundrum2.pdf.

48. Randall, L. Sundrum, R. An alternative to compactification, Phys Rev Let, 1999; 83, 4690-4693.

49. Amoroso, R.L. Unified field mechanics: A brief introduction, R.L. Amoroso, L.H. Kauffman Eds. Unified Field Mechanics: Natural Science Beyond the Veil of Spacetime, 2016; 176-180, World Scientific: London. 
50. Ali, A.F. Faizal, M. Khalil, M.M. Absence of black holes at LHC due to gravity's rainbow, ePhysics Let. B, 2015; 743, 295-300; http://arxiv.org/abs/1410.4765.

51. Cavaglia, M. Das, S. Maartens, R. Will we observe black holes at LHC? Class. Quant. Grav., 2003; 20, L205L212.

52. Ali, A.F. No existence of black holes at LHC due to minimal length in quantum gravity, J. High Energy Phys. 2012; 1209, 067.

53. Hossenfelder, S. Suppressed black hole production from minimal length, Phys. Lett. B, 2004; 598, 92-98.

54. Lustig, E. Weimann, S. Plotnik, Y. Lumer, Y. Bandres, M.A., Szameit, A. Segev, M. Photonic topological insulator in synthetic dimensions. Nature, 2019; 567, 7748, 356-360.

55. Livi, L.F. Cappellini, G. Diem, M. Franchi, L. Clivati, C. Frittelli, M. Fallani, L. Synthetic dimensions and spin-orbit coupling with an optical clock transition. Phys rev let, 2016; 117, 22, 220401.

56. Decca, R.S. López, D. Fischbach, E. Krause, D.E. Measurement of the Casimir force between dissimilar metals. Phys rev let, 2003; 91, 5, 050402; Krause, D.E. Fischbach, E. Isotopic dependence of the Casimir force. Phys Rev Let, 2002; 89(19), 190406.

57. Milton, K. Dimensional and dynamical aspects of the Casimir effect: Understanding the reality and significance of vacuum energy, 2000; arXiv:hep-th/0009173v1.

58. Krause, D.E. Fischbach, E. Searching for extra dimensions and new string-inspired forces in the Casimir regime, 1999; https://arxiv.org/pdf/hep-ph/9912276.pdf.

59. Feynman, R.P. Quantum mechanical computers, Found Phys, 1986; 116, 507.

60. Biafore, M. Can quantum computers have simple Hamiltonians? Proceedings Workshop on Physics and Computation. PhysComp'94, 1994; IEEE, 63-68.

61. Huerta, J. How space-times emerge from the Superpoint, LMS/EPSRC Durham Symposium on Higher Structures in M-Theory, 2019; arXiv:190302822v1 [hep-th].

62. Amoroso, R.L. Rauscher, E.A. Speculation on a unified field theory (UFT) grand unification theories (GUT) supersymmetry and superstring theories, Orbiting the Moons of Pluto: Complex Solutions to the Einstein, Maxwell, Schrodinger, and Dirac Equations, 2011; World Scientific: Singapore, 238-266; (http://vixra.org/pdf/1802.0434v1.pdf.

63. Amoroso, R.L. Buckaroo Banzai across the 8th dimension: A strategic assault on the dimensional barrier Proceedings ANPA 39, 2018; http://anpa.onl/wp-content/uploads/2018/04/Amoroso-ANPA18-Topology.pdf

64. Amoroso, R.L, Millennial science, the imminent age of discovery's conscious technologies, Essays on Consciousness Towards a New Paradigm, I. Fredriksson Ed. 2018; Balboa Press.

65. Luminet, J-P The wraparound universe, 2008; CRC Press.

66. Luminet, J-P Weeks, J.R. Riazuelo, A. Lehoucq, R. Uzan, J-P Dodecahedral space topology as an explanation for weak wide-angle temperature correlations in the cosmic microwave background. Nature, 2003; 425,6958, 593-595.

67. Luminet, J-P The shape and topology of the universe, 2008; arXiv preprint arXiv:0802.2236.

68. Sung, J.C. Pixels of Space-Time, 1993; Woburn Scientific Publications.

69. Comoglio, F. Rinaldi, M. A topological framework for the computation of the homfly polynomial and its application to proteins. PLoS ONE, 2011; 6,4, e18693; https://doi.org/10.1371/journal.pone.0018693.

70. Dabrowski-Tumanski, P._Knots, links, and lassos - Topological manifolds in biological objects,_2019; DOI: 10.13140/RG.2.2.10517.88808, Thesis PhD.

71. Langer J.C. Singer, D.A. The trefoil, Milan J Math, 2013; 99, 9999, 1-23.

72. Quigg, C. The double simplex, 2005; arXiv:hep-ph/0509037.

73. Wolchover, N. Velasco, S. Reading, L. A new map of particles and forces, Quanta Mag., 2020; https://www.quantamagazine.org/a-new-map-of-the-standard-model-of-particle-physics-20201022/.

74. Dirac, P.A.M. Is there an aether? Nature, 1951; 168,4282, 906-907.

75. Hainzl, C. Lewin, M. Séré, É. Existence of a stable polarized vacuum in the Bogoliubov-Dirac-Fock approximation. Communications in mathematical physics, 2005; 257, 3, 515-562; https://arxiv.org/pdf/mathph/0403005.pdf.

76. Lehnert, B., Roy, S. Extended Electromagnetic Theory, Space Charge in Vacuo and Rest Mass of Photon, 1998; 16, World Scientific, London.

77. Lehnert, B. Scheffel, J. On the minimum elementary charge of an extended electromagnetic theory. Physica Scripta, 2002; 65, 3, 200.

78. Vigier, J-P Possible consequences of an extended charged particle model in electromagnetic theory. Physics Letters A, 1997; 235, 5, 419-431.

79. Vigier, J-P Amoroso, R.L. Can one unify gravity and electromagnetic fields? R.L. Amoroso, J-P Vigier Eds. Gravitation and Cosmology: From the Hubble Radius to the Planck Scale, 2002; 241-258. Springer: Dordrecht.

80. Amoroso, R.L. Rowlands, P. Kauffman, L.H. Exploring novel cyclic extensions of Hamilton's dualquaternion algebra, R.L. Amoroso, P. Rowlands Eds. The Physics of Reality: Space, Time, Matter, Cosmos, 
2013; 81-91, World Scientific: London.

81. Lemaître G., Amoroso, R.L. Trans. Quaternions and elliptical space (Quaternions et Espace Elliptique) Proceedings, 10th Symposium Honoring Noted French Mathematical Physicist Jean-Pierre Vigier: Unified Field Mechanics II: Formulations and Empirical Tests, 2018; World Scientific: London, 85-97, DOI: 10.1142/9789813232044_000; https://vixra.org/pdf/1706.0034v1.pdf.

82. Antippa, A.F. Dubois, D.M. The dual incursive system of the discrete harmonic oscillator. D.M. Dubois Ed. AIP Conference Proceedings, 2006; 839, 1, 11-64, American Institute of Physics.

83. Dubois, D.M. Review of incursive, hyperincursive and anticipatory systems-foundation of anticipation in electromagnetism. D.M. Dubois Ed. AIP Conference Proceedings, 2000; 517, 1, 3-30. American Inst Physics.

84. Stoney, G.J. On the physical units of nature, Phil. Mag. 1881; 11, 381-391.

85. Amoroso, R.L. Di Biase, F. Empirical protocols for mediating long-range coherence in biological systems, $J$ Consc Explor Res, 2013; 4, 9, 955-976.

86. Chu, M-Y J. Amoroso R.L. Empirical mediation of the primary mechanism initiating protein conformation in prion propagation, D. Dubois Ed. Partial Proceedings of CASYSO7 IJCAS, 2008; 22, Univ Liege Belgium; http://vixra.org/pdf/1305.0090v1.pdf.

87. Kim, O. Deb, P. Beige, A. Cavity-mediated collective laser-cooling of a non-interacting atomic gas in an asymmetric trap to very low temperatures, 2017; arXiv:1506 02910v5 [quant-ph].

88. Rueda, A. Haisch, B. Contribution to inertial mass by reaction of the vacuum to accelerated motion. Found Phys, 1998; 28, 7, 1057-1108.

89. Rueda, A. Haisch, B. Inertia as reaction of the vacuum to accelerated motion. Phys Let A, 1998; 240, 3, 115126.

90. Rueda, A. Haisch, B. Physics Lett. A, 1998; 240, 115.

91. Rueda, A. Haisch, B. Puthoff, H.E. Phys. Rev. A, 1994; 49, 678.

92. Rueda, A. Haisch, B. The inertia reaction force and its vacuum origin, R.L. Amoroso, G. Hunter, M. Kafatos J-P Vigier Eds. Gravitation \& Cosmology: From the Hubble Radius to the Planck Scale, 2002; 447-458, Kluwer Academic (Elsevier): Dordrecht.

93. Vigier, J-P Derivation of inertial forces from the Einstein-de Broglie-Bohm causal stochastic interpretation of quantum mechanics, Found. Phys, 1995; 25,10, 1461-1494.

94. Sakharov, A.D. Sov. Phys. Dokl. 1968; 12, 1040.

95. Puthoff, H.E. Polarizable vacuum approach to general relativity, R.L. Amoroso, G. Hunter, M. Kafatos, J-P Vigier Eds. Gravitation \& Cosmology: From the Hubble Radius to the Planck Scale, 2002; 431-446, Kluwer Academic (Elsevier): Dordrecht.

96. Burns, J.E. Entropy and vacuum radiation, Found. Phys. 1998; 28, 7, 1191-1207.

97. Burns, J.E. Vacuum radiation, entropy and the arrow of time, R.L. Amoroso, G. Hunter, S. Jeffers \& M. Kafatos, Eds., Gravitation \& Cosmology: From the Hubble Radius to the Planck Scale, 2002; Kluwer Academic (Elsevier): Dordrecht.

98. Zeh, H.D. The Physical Basis of the Direction of Time, 1989; Springer-Verlag: New York.

99. Vigier, J-P Amoroso, R.L. Can one unify gravity and electromagnetic fields? R.L. Amoroso, G. Hunter, S. Jeffers, M. Kafatos, Eds., Gravitation \& Cosmology: From the Hubble Radius to the Planck Scale, 2002; Kluwer Academic (Elsevier): Dordrecht.

100. Elitzur, A.C. Vaidman, L. Quantum mechanical interaction-free measurements. Found Phys, 1993; 23, 987 997.

101. Kwiat, P. Weinfurter, H. Herzog, T. Zeilinger, A. Kasevich, M. Interaction-free quantum measurements. Phys. Rev. Lett. 1995; 74, 4763-4766.

102. du Marchie Van Voorthuysen, E.H. Realization of an interaction-free measurement of the presence of an object in a light beam. Am. J. Phys. 1996; 64, 12; 1504-1507; arXiv:quant-ph/9803060 v2 26.

103. Simon, S.H. Platzman, P.M. Fundamental limit on "interaction free" measurements, 1999; arXiv:quantph/9905050v1.

104. Vaidman, L. Interaction-free measurements, 1996; arXiv:quant-ph/9610033v1.

105. Paraoanu, G.S. Interaction-free measurements with superconducting qubits, Phys Rev. Lett, 2006; 97, 180406.

106. Vaidman, L. The meaning of the interaction-free measurements, 2001; arXiv:quant-ph/0103081v1.

107. Gordon, J. Rucker, R. Walker, J. James Gleick's Chaos: The Software, Version 1.0, 1990; Autodesk: Sausalito; http://www.rudyrucker.com/oldhomepage/chaos.htm.

108. Hocking, J.G. Young, G.S. Topology, 1988; Dover: New York.

109. Klimchitskaya, G.L. Mostepanenko, V.M. Casimir and van der Waals forces advances and problems, 2015; https://arxiv.org/ftp/arxiv/papers/1507/1507.02393.pdf.

110. van Oss, C.J. Absolom, D.R. Neumann, A.W. Applications of net repulsive van der Waals forces between different particles macro-molecules or biological cells, Liquids Colloids and Surfaces, 1980; 1, 1, 45-56. 
111. Shifman, M. Large extra dimensions: becoming acquainted with an alternative paradigm. Intl J Mod Phys A, 2010; 25, 2, 3, 199-225; arXiv:0907.3074.

112. Weinberg, S. A Model of Leptons, Phys. Rev. Lett, 1967; 19, 21, 1264-66.

113. Gomez-Bock, M. et al. Electroweak symmetry breaking and Higgs physics: basic concepts, J Phys Conf Ser, 2005; 18, 002.

114. Glashow, S. The renormalizability of vector meson interactions, Nucl. Phys, 1959; 10, 107.

115. Salam, A. Ward, J.C. Weak and electromagnetic interactions, Nuovo Cimento, 1959; 11, 4, 568-577.

116. Lee, T.D. Particle physics and introduction to field theory; Contemporary Concepts in Physics, 1981; 1 , Harwood Academic.

117. Vigier, J-P. An electromagnetic theory of strong interactions, Phys Let A, 2003; 319, 3-4, 246-250.

118. Amoroso, R.L. Giandinoto, S. Karam, S.E. Wildfire suppression technology: Exploration for a directed energy beam (DEB) attenuating electron transfer by cyclical vacuum subduction of dioxygen dication species, $\mathrm{O}_{22} 2^{+}$, Journal Physics: Conf Series, 2019; 1251, 1, 012006, IOP Publishing.

119. Moon, J. Executive Intelligence Review, 1987; 18, 6 Nov.

120. Zilner, E. Ciardiello, L. Metatron's cube and the Platonic solids, 2015; http://demonstrations. wolfram.com/MetatronsCubeAndThePlatonicSolids/.

121. Gross, D. Personal communication during presentation: Intl Conf on 60 Years of Yang-Mills Gauge Field Theories, 25-28 May 2015; Nanyang Technological University: Singapore.

122. Hossenfelder, S. Why the foundations of physics have not progressed for 40 years, iai News, 2020; Iss. 84, 8th January; https://iai.tv/articles/why-physics-has-made-no-progress-in-50-years-auid-1292.

123. Feynman, R.P. Feynman Lectures on Gravitation, 2018; CRC Press: Boca Raton.

124. Penrose, R. Nobel prize in physics, (2020; https://physicsworld.com/a/roger-penrose-discusses-his-career-inmathematical-physics/. 